\title{
A CENTRAL LIMIT THEOREM FOR INCOMPLETE U-STATISTICS OVER TRIANGULAR ARRAYS
}

\author{
MATTHIAS LÖWE AND SARA TERVEER
}

\begin{abstract}
We analyze the fluctuations of incomplete $U$-statistics over a triangular array of independent random variables. We give criteria for a Central Limit Theorem (CLT, for short) to hold in the sense that we prove that an appropriately scaled and centered version of the U-statistic converges to a normal random variable. Our method of proof relies on a martingale CLT. A possible application - a CLT for the hitting time for random walk on random graphs - will be presented in LT20.
\end{abstract}

\section{INTRODUCTION}

U-statistics constitute a general method to construct unbiased minimum variance estimators in the theory of statistics. A thorough investigation of their properties can be found, e.g., in the monographs [Den85] or [Lee90]. U-statistics also naturally appear in other contexts, like in the theory of random graphs where they count occurrences of certain subgraphs, e.g. triangles (cf. [Jan90]). In the latter case the U-statistics are incomplete, by which we mean that not all possible combinations of the random variables are taken into account. Such a "dilution" can also be random, as considered in [Jan84]. After having established a law of large numbers for Ustatistics (cf. Chr92]) the most obvious next question is to analyze their asymptotic distribution. This was already investigated in a seminal paper by Hoeffding [Hoe48]. In general, whether a U-statistic is asymptotically normal or not, may depend on whether its kernel function is degenerate or not ([Den85]), i.e. on whether the conditional expectation of the kernel function given some the variables is zero or not. Berry-Esseen theorems and Edgeworth expansions around this CLT were analyzed, among others, in [BG95] and BGvZ86]. Fluctuation results for U-statistics on the level of large or moderate deviations were obtained in [EL95, [EL98], and Eic98]. In this note we will study a situation where the random variables in the U-statistic stem from a triangular array as in MA87. However, additionally to this we consider incomplete U-statistics, where a random variable determines whether a certain summand is taken into account or not. Finally, also the kernel function $h$ may change

2010 Mathematics Subject Classification. 60B20, 05C81, 05C80.

Key words and phrases. Central Limit Theorem, U-statistics, incomplete U-statistics, triangular arrays.

Research of both authors was funded by the Deutsche Forschungsgemeinschaft (DFG, German Research Foundation) under Germany's Excellence Strategy EXC 2044-390685587, Mathematics Münster: Dynamics-Geometry-Structure. 
with $n$, the line number of the triangular array. This situation is motivated by our analysis of hitting times for random walks on random graphs in an accompanying paper (see [LT20]). However, as this situation also is a generalization of the settings in [Jan84, JJJ86], and [MA87], we think it might be also interesting in its own rights. To be more precise, let us describe our setting formally. Let $X_{n 1}, \ldots, X_{n n}, n=$ $1,2, \ldots, \infty$ be a triangular array of random variables with values in some measurable space (for the sake of this paper this measurable space will be $\mathbb{R}$ ), independent of each other and having the same distribution function $F_{n}(x)$ in each row. Let $h_{n}: \mathbb{R} \times \mathbb{R} \rightarrow \mathbb{R}$ be a real-valued, symmetric Borel function. For $i, j=1, \ldots, n$, let

$$
\Phi_{n}(i, j)=Z_{i j} \cdot h_{n}\left(X_{n i}, X_{n j}\right)
$$

(of course, $\Phi_{n}$ is a function of $Z_{i j}, X_{n i}$, and $X_{n j}$ rather than just of $i, j$; however, for the sake of brevity we will omit the variables here and in the following definitions). Here the $Z_{i j}=Z_{j i}$ are assumed to be i.i.d. $\operatorname{Ber}\left(p_{n}\right)$ random variables (apart from the constraint that $Z_{i j}=Z_{j i}$ ) that are independent of the triangular array of the $\left(X_{n i}\right)$. Throughout this note we will assume that $p$ may depend on $n$, but that $n p \rightarrow \infty$. Moreover, assume that for all $n \in \mathbb{N}$ and $1 \leq i \neq j \leq n$

$$
\mathbb{E}\left[h_{n}\left(X_{n i}, X_{n j}\right)\right]=0 \quad \text { and } \quad \mathbb{E}\left[h_{n}^{2}\left(X_{n i}, X_{n j}\right)\right]<\infty .
$$

Let us construct the following U-statistic

$$
\mathcal{U}_{n}=\left(\begin{array}{l}
n \\
2
\end{array}\right)^{-1} \sum_{1 \leq i<j \leq n} \Phi_{n}(i, j)=\left(\begin{array}{l}
n \\
2
\end{array}\right)^{-1} \sum_{1 \leq i<j \leq n} Z_{i, j} \cdot h_{n}\left(X_{n i}, X_{n j}\right) .
$$

To construct a Hoeffding-type decomposition (see [Hoe48]) we introduce

$$
\begin{gathered}
\Psi_{j}^{(n)}(i):=\mathbb{E}\left[\Phi_{n}(i, j) \mid X_{i}, Z_{i j}\right]=Z_{i j} \mathbb{E}\left[h_{n}\left(X_{n i}, X_{n j}\right) \mid X_{n i}\right] \\
\beta_{n}^{2}:=\mathbb{E}\left[\Phi_{n}^{2}(1,2)\right], \quad \gamma_{n}^{2}:=\mathbb{E}\left[\left(\Psi_{2}^{(n)}(1)\right)^{2}\right], \text { and } \theta_{n}^{2}:=n p_{n} \gamma_{n}^{2}+\beta_{n}^{2} / 2 .
\end{gathered}
$$

Then obviously, $\Phi_{n}(i, j)$ and $\Psi_{j}^{(n)}(i)$ are centered. Next, put

$$
\begin{aligned}
\tilde{\Phi}_{n}(i, j) & =\Phi_{n}(i, j)-\Psi_{j}^{(n)}(i)-\Psi_{i}^{(n)}(j), \\
\tilde{h}_{n}\left(X_{n i}, X_{n j}\right) & =h_{n}\left(X_{n i}, X_{n j}\right)-\mathbb{E}\left[h_{n}\left(X_{n i}, X_{n j}\right) \mid X_{n i}\right]-\mathbb{E}\left[h_{n}\left(X_{n i}, X_{n j}\right) \mid X_{n j}\right] .
\end{aligned}
$$

Then $\tilde{\Phi}_{n}(i, j)=Z_{i, j} \tilde{h}_{n}\left(X_{n i}, X_{n j}\right)$ is again centered - we even have for every $i \neq j$ and every $k$

$$
\mathbb{E}\left[\tilde{h}_{n}\left(X_{n i}, X_{n j}\right) \mid X_{n k}\right]=0
$$

even for $k=i, j$ (as can be seen by applying the definition of $\tilde{h}_{n}$ ).

In the following we omit the index $n$ whenever suitable. We will also frequently write $h(i, j)$ and $\tilde{h}(i, j)$ as shorthand notations for $h_{n}\left(X_{n i}, X_{n j}\right)$ and $\tilde{h}_{n}\left(X_{n i}, X_{n j}\right)$. Let us collect some properties of the above quantities in the following lemma, whose proof is deferred to the appendix:

Lemma 1.1. For any $i \neq j$ we have: 
(1) $\mathbb{E}\left[\tilde{\Phi}^{2}(i, j)\right]=\beta_{n}^{2}-2 \gamma_{n}^{2}$.

(2) $\mathbb{E}\left[\mathbb{E}\left[h(i, j) \mid X_{i}\right]^{2}\right]=\frac{\gamma_{n}^{2}}{p}, \mathbb{E}\left[h^{2}(i, j)\right]=\frac{\beta_{n}^{2}}{p}$ and $\mathbb{E}\left[\tilde{h}(i, j)^{2}\right]=\frac{\beta_{n}^{2}-2 \gamma_{n}^{2}}{p}$.

We can now compute the Hoeffing decomposition of $\mathcal{U}_{n}$ (again the proof is given in the appendix):

Lemma 1.2. We can rewrite $\mathcal{U}_{n}$ in the following way:

$$
\mathcal{U}_{n}=\sum_{i=1}^{n}\left(\left(\begin{array}{l}
n \\
2
\end{array}\right)^{-1} \sum_{j=1}^{i-1} \tilde{\Phi}(i, j)+\left(\begin{array}{l}
n \\
2
\end{array}\right)^{-1} \sum_{\substack{j=1 \\
j \neq i}}^{n} \Psi_{j}(i)\right)
$$

This allows to compute the asymptotic variance of $\mathcal{U}_{n}$. We will show in the appendix

Lemma 1.3. For the variance of $\mathcal{U}_{n}$ we have the following asymptotic identities

$$
\mathbb{V} \mathcal{U}_{n} \sim\left(\begin{array}{l}
n \\
2
\end{array}\right)^{-1}\left(\beta_{n}^{2}+2 n p \gamma_{n}^{2}\right)=\left(\begin{array}{l}
n \\
2
\end{array}\right)^{-1} 2 \theta_{n}^{2} \text { and } \sqrt{\mathbb{V} \mathcal{U}_{n}}\left(\begin{array}{l}
n \\
2
\end{array}\right) \sim n \theta_{n}
$$

Here and below, for two sequences $\left(a_{n}\right)$ and $\left(b_{n}\right)$ we write $a_{n} \sim b_{n}$, if $a_{n} / b_{n} \rightarrow 1$. To prove a CLT for $\mathcal{U}_{n}$ we will consider

$$
\frac{\mathcal{U}_{n}}{\sqrt{\mathbb{V} \mathcal{U}_{n}}} \sim \sum_{i=1}^{n} \xi_{i}
$$

with $\xi_{i, n}=\xi_{i}=\xi_{i}^{(1)}+\xi_{i}^{(2)}$ for $i=1, \ldots, n$, where

$$
\xi_{i}^{(1)}=\xi_{i, n}^{(1)}=\frac{1}{n \theta_{n}} \sum_{\substack{j=1 \\ j \neq i}}^{n} \Psi_{j}(i), \quad \xi_{i}^{(2)}=\xi_{i, n}^{(2)}=\frac{1}{n \theta_{n}} \sum_{j=1}^{i-1} \tilde{\Phi}(i, j) .
$$

We are aiming to prove the following results:

Theorem 1.4. Assume that for all $\varepsilon>0$

$$
\begin{gathered}
\eta_{1}=\sum_{i=1}^{n} \mathbb{E}\left[\xi_{i}^{2} \mathbb{1}_{\left\{\left|\xi_{i}\right| \geq \varepsilon\right\}} \mid\left(X_{k}\right)_{k=1, \ldots, i-1},\left(Z_{l, m}\right)_{\substack{l=1, \ldots, i-1, m=1, \ldots, n, m \neq l}}\right] \underset{n \rightarrow \infty}{\stackrel{\mathbb{P}}{\longrightarrow}} 0, \\
\eta_{2}=\sum_{i=1}^{n} \mathbb{E}\left[\xi_{i}^{2} \mid\left(X_{k}\right)_{k=1, \ldots, i-1},\left(Z_{l, m}\right)_{\substack{l=1, \ldots, i-1, m=1, \ldots, n, m \neq l}} \underset{n \rightarrow \infty}{\stackrel{\mathbb{P}}{\longrightarrow}} 1 .\right.
\end{gathered}
$$

Then $\frac{\mathcal{U}_{n}}{\sqrt{\mathbb{V U _ { n }}}}$ converges in distribution to a standard normal random variable.

To give alternative conditions that will be useful in the application we have in mind let us introduce

$$
\begin{aligned}
& G_{k}(i, j)=\mathbb{E}\left[\Phi(i, k) \Phi(j, k) \mid X_{i}, X_{j}, Z_{i k}, Z_{j k}\right]=: Z_{i k} Z_{j k} H(i, j) \\
& \tilde{G}_{k}(i, j)=\mathbb{E}\left[\tilde{\Phi}(i, k) \tilde{\Phi}(j, k) \mid X_{i}, X_{j}, Z_{i k}, Z_{j k}\right]=: Z_{i k} Z_{j k} \tilde{H}(i, j) .
\end{aligned}
$$

Then, 
Theorem 1.5. Assume that for all $\varepsilon>0$

$$
\begin{array}{r}
\frac{1}{n \theta_{n}^{2}} \mathbb{E}\left[\left(\sum_{j=2}^{n} \Psi_{j}(1)\right)^{2} \mathbb{1}_{\left\{\left|\sum_{j=2}^{n} \Psi_{j}(1)\right| \geq \varepsilon \theta_{n} n\right\}} \stackrel{n \rightarrow \infty}{\longrightarrow} 0\right. \\
\theta_{n}^{-2} \mathbb{E}\left[\tilde{\Phi}^{2}(1,2) \mathbb{1}_{\left\{|\tilde{\Phi}(1,2)| \geq \varepsilon \theta_{n} n\right\}} \stackrel{n \rightarrow \infty}{\longrightarrow} 0\right. \\
p \theta_{n}^{-2} \mathbb{E}\left[\tilde{H}(1,1) \mathbb{1}_{\left\{|\tilde{H}(1,1)| \geq \frac{\varepsilon \theta_{n}^{2} n}{p}\right\}} \stackrel{n \rightarrow \infty}{\longrightarrow} 0\right. \\
\theta_{n}^{-4} \mathbb{E}\left[G_{1}^{2}(2,3)\right] \stackrel{n \rightarrow \infty}{\longrightarrow} 0
\end{array}
$$

Then again $\frac{\mathcal{U}_{n}}{\sqrt{\sqrt{\mathcal{U}_{n}}}}$ converges in distribution to a standard normal random variable.

Remark 1.6. It is well known that there are situations where $\mathcal{U}_{n}$ does not obey a CLT. These are for example situations without dilution, e.g. when the $Z_{i j} \equiv 1$ with probability 1, and if the kernel function $h=h_{n}$ is degenerate, i.e. when $\mathbb{E}[h(X, Y) \mid$ $Y]=0$ for independent random variables $X, Y$ with the same distribution as $X_{11}$ (for the time being we assume that the distribution of $X_{n i}$ does not depend on $n$ and $i$, so we simply have a sequence of i.i.d. random variables $\left.X_{1}, X_{2}, \ldots\right)$.

For a typical situations consider $\mathbb{P}\left(Z_{i j}=1\right)=1$ and $h\left(X_{1}, X_{2}\right)=X_{1} X_{2}$. If then, $\mathbb{E} X_{1}=0$ and $\mathbb{E} X_{1}^{2}=1$, the rescaled $U$-statistic $n \mathcal{U}_{n}$ does not converge to a normally distributed random variable but to a $\chi_{1}^{2}$-random variable. This can be seen by the CLT together with an application of the continuous mapping theorem. In this situation we quickly check that also condition (C4) breaks down. Indeed, one checks that $\theta_{n}^{2}=\frac{1}{2}$, since $\beta_{n}^{2}=1$, and that $\gamma_{n}^{2}=0$. Moreover,

$$
G_{1}(2,3)=H(2,3)=\mathbb{E}\left[h(1,2) h(1,3) \mid X_{2}, X_{3}\right]=X_{2} X_{3} \mathbb{E}\left[X_{1}^{2}\right]=X_{2} X_{3}
$$

(recall that $\left.Z_{i, j} \equiv 1\right)$. This means for (대 that

$$
\theta_{n}^{-4} \mathbb{E}\left[G_{1}^{2}(2,3)\right]=4 \cdot \mathbb{E}\left[X_{2}^{2} X_{3}^{2}\right]=4 \mathbb{E}\left[X_{2}^{2}\right] \mathbb{E}\left[X_{3}^{2}\right]=4,
$$

which does not go to 0 . Hence (C4) is violated.

The rest of this note is organized in the following way. In Section 2 we will prove Theorem 1.4. A main ingredient to this end will be a martingale CLT due to Girko (see Theorem 2.1 below). In Section 3 we will prepare for the proof of Theorem 1.5 by giving an alternative condition for (C4) (see condition ( $\overline{\mathrm{C} 4}$ ' below). The core of the paper is the proof of Theorem 1.5 in Section 4. We will see that (C1)-(C4) (resp. (C4) ) imply the conditions of Theorem 1.4. Finally, in Section 5, we will give some alternative conditions for (C1)-(C3) that are easier to check in some applications. The appendix contains the proofs of our technical results.

\section{Proof of Theorem 1.4}

As mentioned in the Introduction we will base our arguments on the following theorem ([Gir90, Theorem 5.4.11]): 
Theorem 2.1. Consider a triangular array of martingale differences $\left(Y_{i, n}\right), i=$ $1, \ldots, n$ and a sequence of filtrations $\left(\mathcal{F}_{i, n}\right)_{i=1, \ldots, n}$. If for any $\varepsilon>0$

$$
\sum_{i=1}^{n} \mathbb{E}\left[Y_{i, n}^{2} \mathbb{1}_{\left\{\left|Y_{i, n}\right| \geq \varepsilon\right\}} \mid \mathcal{F}_{i-1, n}\right] \underset{n \rightarrow \infty}{\stackrel{\mathbb{P}}{\longrightarrow}} 0 \text { and } \sum_{i=1}^{n} \mathbb{E}\left[Y_{i, n}^{2} \mid \mathcal{F}_{i-1, n}\right] \underset{n \rightarrow \infty}{\stackrel{\mathbb{P}}{\longrightarrow}} 1
$$

hold, then $\sum_{i=1}^{n} Y_{i, n}$ converges in distribution to a standard normal random variable.

To apply this result let $\mathcal{F}_{i}=\mathcal{F}_{i, n}=\sigma\left(\left(X_{k}\right)_{k=1, \ldots, i},\left(Z_{l, m}\right)_{l=1, \ldots, i,} \begin{array}{l}m=1, \ldots, n, m \neq l \\ m\end{array}\right)$. Using the notation from Section $1, \Psi_{j}(i)$ is $\mathcal{F}_{i}$-measurable, hence $\xi_{i}^{(1)}$ is $\mathcal{F}_{i}$-adapted. For $j<i$, $X_{j}$ is also $\mathcal{F}_{i}$-measurable, so that $\tilde{\Phi}(i, j)$ and therefore $\xi_{i}^{(2)}$ are also $\mathcal{F}_{i}$-adapted. Hence $\xi_{i}$ is $\mathcal{F}_{i}$-adapted. Now,

$$
\begin{gathered}
\mathbb{E}\left[\xi_{i} \mid \mathcal{F}_{i-1}\right]=\frac{1}{n \theta_{n}}\left(\sum_{j<i} \mathbb{E}\left[Z_{i j} \mathbb{E}\left[h(i, j) \mid X_{i}\right] \mid \mathcal{F}_{i-1}\right]+\sum_{j>i} \mathbb{E}\left[Z_{i j} \mathbb{E}\left[h(i, j) \mid X_{i}\right] \mid \mathcal{F}_{i-1}\right]\right. \\
\left.+\sum_{j<i} \mathbb{E}\left[Z_{i j} \tilde{h}(i, j) \mid \mathcal{F}_{i-1}\right]\right)
\end{gathered}
$$

By definition, for $j<i, Z_{i j}=Z_{j i}$ are $\mathcal{F}_{i-1}$-measurable, while $X_{i}$ is independent of $\mathcal{F}_{i-1}$. For the second term notice that both $X_{i}$ and $Z_{i j}$ are independent of $\mathcal{F}_{i-1}$ in the case $j>i$. In the third sum, the $Z_{i, j}$ is measurable again. This leaves only $\tilde{h}(i, j)$, which is independent of all but one condition: $X_{j}$. Therefore

$$
\begin{aligned}
& \mathbb{E}\left[\xi_{i} \mid \mathcal{F}_{i-1}\right] \\
& =\frac{1}{n \theta_{n}}\left(\sum_{j<i} Z_{i j} \mathbb{E}[h(i, j)]+\sum_{j>i} \mathbb{E}\left[Z_{i j}\right] \mathbb{E}[h(i, j)]+\sum_{j<i} Z_{i j} \mathbb{E}\left[\tilde{h}(i, j) \mid X_{j}\right]\right)=0
\end{aligned}
$$

by (1.1) and (1.4). Thus $\xi_{i}$ is a martingale difference. Setting $Y_{i}=Y_{i, n}=\xi_{i, n}$ in Theorem 2.1 we can rewrite conditions a) and b) in this theorem as

$$
\begin{aligned}
& \eta_{1}=\sum_{i=1}^{n} \mathbb{E}\left[\xi_{i}^{2} \mathbb{1}_{\left\{\left|\xi_{i}\right| \geq \varepsilon\right\}} \mid\left(X_{k}\right)_{k=1, \ldots, i-1},\left(Z_{l, m}\right)_{\substack{l=1, \ldots, i-1, m=1, \ldots, n, m \neq l}}\right] \underset{n \rightarrow \infty}{\stackrel{\mathbb{P}}{\longrightarrow}} 0, \\
& \eta_{2}=\sum_{i=1}^{n} \mathbb{E}\left[\xi_{i}^{2} \mid\left(X_{k}\right)_{k=1, \ldots, i-1},\left(Z_{l, m}\right)_{\substack{l=1, \ldots, i-1, m=1, \ldots, n, m \neq l}}\right] \underset{n \rightarrow \infty}{\stackrel{\mathbb{P}}{\longrightarrow}} 1 .
\end{aligned}
$$

This proves Theorem 1.4.

\section{An Alternative CONDition FOR (C4)}

The purpose of this section is to prove

Proposition 3.1. (C4) implies

$$
\theta_{n}^{-4} \mathbb{E}\left[\tilde{G}_{1}^{2}(2,3)\right] \stackrel{n \rightarrow \infty}{\longrightarrow} 0 .
$$


Proof. For $i, j, k$ pairwise different, we have by definition of $\tilde{G}_{k}$

$$
\begin{aligned}
& \tilde{G}_{k}(i, j)=\mathbb{E}\left[\Phi(i, k) \Phi(j, k)-\Psi_{k}(i) \Phi(j, k)-\Psi_{i}(k) \Phi(j, k)-\Psi_{k}(j) \Phi(i, k)-\Psi_{j}(k) \Phi(i, k)\right. \\
& \left.\quad+\Psi_{k}(i) \Psi_{k}(j)+\Psi_{k}(i) \Psi_{j}(k)+\Psi_{i}(k) \Psi_{k}(j)+\Psi_{i}(k) \Psi_{j}(k) \mid X_{i}, X_{j}, Z_{i, k}, Z_{j, k}\right] \\
& =G_{k}(i, j)-\Psi_{k}(i) \Psi_{k}(j)-\mathbb{E}\left[\Psi_{i}(k) \Phi(j, k) \mid X_{i}, X_{j}, Z_{i, k}, Z_{j, k}\right]-\Psi_{k}(i) \Psi_{k}(j) \\
& \quad-\mathbb{E}\left[\Psi_{j}(k) \Phi(i, k) \mid X_{i}, X_{j}, Z_{i, k}, Z_{j, k}\right]+\Psi_{k}(i) \Psi_{k}(j)+\Psi_{k}(i) \mathbb{E}\left[\Psi_{j}(k) \mid X_{i}, X_{j}, Z_{i, k}, Z_{j, k}\right] \\
& \quad+\Psi_{k}(j) \mathbb{E}\left[\Psi_{i}(k) \mid X_{i}, X_{j}, Z_{i, k}, Z_{j, k}\right]+\mathbb{E}\left[\Psi_{i}(k) \Psi_{j}(k) \mid X_{i}, X_{j}, Z_{i, k}, Z_{j, k}\right]
\end{aligned}
$$

Now $\mathbb{E}\left[\Psi_{j}(k) \mid X_{i}, X_{j}, Z_{i, k}, Z_{j, k}\right]=0$, and three of the above terms only differ by their sign. Thus

$$
\begin{aligned}
& \tilde{G}_{k}(i, j)=G_{k}(i, j)-\Psi_{k}(i) \Psi_{k}(j)-\mathbb{E}\left[\Psi_{i}(k) \Phi(j, k) \mid X_{i}, X_{j}, Z_{i, k}, Z_{j, k}\right] \\
& \quad-\mathbb{E}\left[\Psi_{j}(k) \Phi(i, k) \mid X_{i}, X_{j}, Z_{i, k}, Z_{j, k}\right]+\mathbb{E}\left[\Psi_{i}(k) \Psi_{j}(k) \mid X_{i}, X_{j}, Z_{i, k}, Z_{j, k}\right] \\
& =: G_{k}(i, j)-\Psi_{k}(i) \Psi_{k}(j)-A-B+C .
\end{aligned}
$$

By independence, $\mathbb{E}\left[\left(\Psi_{k}(i) \Psi_{k}(j)\right)^{2}\right]=\mathbb{E}\left[\Psi_{k}^{2}(i)\right] \mathbb{E}\left[\Psi_{k}^{2}(j)\right]=\gamma_{n}^{4}$, and for $A$, we have again by independence and Cauchy-Schwarz

$$
\begin{aligned}
\mathbb{E}\left[A^{2}\right] & =\mathbb{E}\left[Z_{i, k} Z_{j, k}\left(\mathbb{E}\left[\mathbb{E}\left[h(i, k) \mid X_{k}\right] h(j, k) \mid X_{j}\right]\right)^{2}\right] \\
& \leq p^{2} \mathbb{E}\left[\mathbb{E}\left[\mathbb{E}\left[h(i, k) \mid X_{k}\right]^{2} \mid X_{j}\right] \mathbb{E}\left[h(j, k)^{2} \mid X_{j}\right]\right] \\
& =p^{2} \mathbb{E}\left[\mathbb{E}\left[\mathbb{E}\left[h(i, k) \mid X_{k}\right]^{2}\right] \mathbb{E}\left[h^{2}(j, k) \mid X_{j}\right]\right]
\end{aligned}
$$

and by Lemma 1.1 this equals

$$
=p^{2} \mathbb{E}\left[\frac{\gamma_{n}^{2}}{p} \mathbb{E}\left[h^{2}(j, k) \mid X_{j}\right]\right]=p^{2} \frac{\gamma_{n}^{2}}{p} \mathbb{E}\left[h^{2}(j, k)\right]=p^{2} \frac{\gamma_{n}^{2}}{p} \frac{\beta_{n}^{2}}{p}=\beta_{n}^{2} \gamma_{n}^{2} .
$$

$B^{2}$ has the same upper bound for the expectation, which can be proven analogously. As for $C$, we again use measurability and independence to obtain

$$
\begin{aligned}
\mathbb{E}\left[C^{2}\right] & =\mathbb{E}\left[\mathbb{E}\left[\Psi_{i}(k) \Psi_{j}(k) \mid X_{i}, X_{j}, Z_{i, k}, Z_{j, k}\right]^{2}\right]=\mathbb{E}\left[\mathbb{E}\left[\Psi_{i}(k) \Psi_{j}(k) \mid Z_{i, k}, Z_{j, k}\right]^{2}\right] \\
& =\mathbb{E}\left[Z_{i, k} Z_{j, k} \mathbb{E}\left[\mathbb{E}\left[h(i, k) \mid X_{k}\right] \mathbb{E}\left[h(j, k) \mid X_{k}\right]\right]^{2}\right] \\
& \leq p^{2} \mathbb{E}\left[\mathbb{E}\left[h(i, k) \mid X_{k}\right]^{2}\right]^{2}=p^{2}\left(\frac{\gamma_{n}^{2}}{p}\right)^{2}=\gamma_{n}^{4}
\end{aligned}
$$

by Cauchy-Schwarz and Lemma 1.1 ,

We can combine all this to conclude

$$
\begin{aligned}
\frac{1}{\theta_{n}^{4}} \mathbb{E}\left[\tilde{G}_{1}^{2}(2,3)\right] & \leq \frac{1}{\theta_{n}^{4}} \mathbb{E}\left[\left(G_{1}(2,3)-\Psi_{1}(2) \Psi_{1}(3)-A-B+C\right)^{2}\right] \\
& \leq \frac{25}{\theta_{n}^{4}}\left(\mathbb{E}\left[G_{1}^{2}(2,3)\right]+\mathbb{E}\left[\Psi_{1}^{2}(2) \Psi_{1}^{2}(3)\right]+\mathbb{E}\left[A^{2}\right]+\mathbb{E}\left[B^{2}\right]+\mathbb{E}\left[C^{2}\right]\right) \\
& \leq \frac{25}{\theta_{n}^{4}} \mathbb{E}\left[G_{1}^{2}(2,3)\right]+\frac{25}{\theta_{n}^{4}} 2 \gamma_{n}^{4}+\frac{25}{\theta_{n}^{4}} 2 \beta_{n}^{2} \gamma_{n}^{2} \leq \frac{25}{\theta_{n}^{4}} \mathbb{E}\left[G_{1}^{2}(2,3)\right]+\frac{50}{(n p)^{2}}+\frac{50}{n p}
\end{aligned}
$$


due to (1.3),$\theta_{n}^{2} \geq n p \gamma_{n}^{2}$ and $\theta_{n}^{2} \geq \beta_{n}^{2}$. The second and third term go to 0 as $n p \rightarrow \infty$ for $n \rightarrow \infty$. The first term is exactly the term from (C4), which is assumed to converge to 0 as well. This completes the proof.

\section{Proof of Theorem 1.5}

The proof of Theorem 1.5 immediately follows from

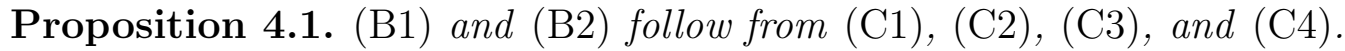

Remark 4.2. Conditions (C1) (C4) may be tricky to check. In fact, in many settings it may be unreasonable to prove conditions for $\tilde{\Phi}$ instead of $\Phi$ etc. In Proposition 5.1 below we will give alternative, more straightforward conditions for (C1) - (C3)

We split the proof of Proposition 4.1 into two Lemmas:

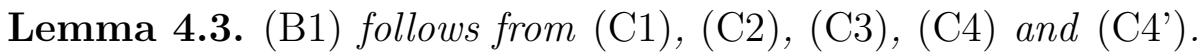

Proof. Since $\eta_{1}$ is non-negative, by Markov's inequality, it suffices to show $\mathbb{E}\left[\eta_{1}\right] \stackrel{n \rightarrow \infty}{\longrightarrow}$ 0 to obtain $\eta_{1} \underset{n \rightarrow \infty}{\stackrel{\mathbb{P}}{\longrightarrow}} 0$. Using Lemma A.8 with $k=2, a_{1}=\xi_{i}^{(1)}$, and $a_{2}=\xi_{i}^{(2)}$ we get

$$
\begin{aligned}
& \eta_{1} \leq 4 \sum_{i=1}^{n}\left(\mathbb{E}\left[\left(\xi_{i}^{(1)}\right)^{2} \mathbb{1}_{\left\{\left|\xi_{i}^{(1)}\right| \geq \frac{\varepsilon}{2}\right\}} \mid\left(X_{k}\right)_{k=1, \ldots, i-1},\left(Z_{l, m}\right)_{\substack{l=1, \ldots, i-1, m=1, \ldots, n, m \neq l}}\right]\right. \\
& \left.+\mathbb{E}\left[\left(\xi_{i}^{(2)}\right)^{2} \mathbb{1}_{\left\{\left|\xi_{i}^{(2)}\right| \geq \frac{\varepsilon}{2}\right\}} \mid\left(X_{k}\right)_{k=1, \ldots, i-1},\left(Z_{l, m}\right)_{\substack{l=1, \ldots, i-1, m=1, \ldots, n, m \neq l}}\right]\right),
\end{aligned}
$$

and consequently,

$$
\begin{aligned}
\mathbb{E}\left[\eta_{1}\right] \leq & \left.4 \sum_{i=1}^{n} \mathbb{E}\left[\left(\frac{1}{n \theta_{n}} \sum_{\substack{j=1 \\
j \neq i}}^{n} \Psi_{j}(i)\right)^{2} \mathbb{1}_{\left\{\mid \frac{1}{n \theta_{n}}\right.} \sum_{j=1, j \neq i}^{n} \Psi_{j}(i) \mid \geq \frac{\varepsilon}{2}\right\}\right] \\
& +4 \sum_{i=1}^{n} \mathbb{E}\left[\left(\frac{1}{n \theta_{n}} \sum_{j=1}^{i-1} \tilde{\Phi}(i, j)\right)^{2} \mathbb{1}_{\left\{\left|\frac{1}{n \theta_{n}} \sum_{j=1}^{i-1} \tilde{\Phi}(i, j)\right| \geq \frac{\varepsilon}{2}\right\}}\right]=: S_{1}+T_{1} .
\end{aligned}
$$

Now,

$S_{1}=\frac{4}{n^{2} \theta_{n}^{2}} \sum_{i=1}^{n} \mathbb{E}\left[\left(\sum_{j=1, j \neq i}^{n} \Psi_{j}(i)\right)^{2} \mathbb{1}_{\left\{\left|\sum_{j=1, j \neq i}^{n} \Psi_{j}(i)\right| \geq \frac{\varepsilon \theta_{n} n}{2}\right\}}\right]=\frac{4}{n \theta_{n}^{2}} \mathbb{E}\left[\left(\sum_{j=2}^{n} \Psi_{j}(1)\right)^{2} \mathbb{1}_{\left.\left\{\left|\sum_{j=2}^{n} \Psi_{j}(1)\right| \geq \frac{\varepsilon \theta_{n} n}{2}\right\}\right]}\right]$

by identical distribution. Hence, by (C1), we have $S_{1} \stackrel{n \rightarrow \infty}{\longrightarrow} 0$. 
The estimate for $T_{1}$ is slightly longer:

$$
\begin{aligned}
& T_{1}=\frac{4}{n^{2} \theta_{n}^{2}} \sum_{i=1}^{n} \mathbb{E}\left[\left(\sum_{j=1}^{i-1} \tilde{\Phi}(i, j)\right)^{2} \mathbb{1}\left\{\left|\sum_{j=1}^{i-1} \tilde{\Phi}(i, j)\right| \geq \frac{\varepsilon \theta_{n} n}{2}\right\}\right] \\
& =\frac{4}{n^{2} \theta_{n}^{2}} \sum_{i=1}^{n} \sum_{j=1}^{i-1} \mathbb{E}\left[\tilde{\Phi}^{2}(i, j) \mathbb{1}_{\left\{\left|\sum_{k=1}^{i-1} \tilde{\Phi}(i, k)\right| \geq \frac{\varepsilon \theta_{n} n}{2}\right\}} \mathbb{1}_{\left.\left\{|\tilde{\Phi}(i, j)| \geq \frac{\varepsilon \theta_{n} n}{4}\right\}\right]}\right. \\
& +\frac{4}{n^{2} \theta_{n}^{2}} \sum_{i=1}^{n} \sum_{j=1}^{i-1} \mathbb{E}\left[\tilde{\Phi}^{2}(i, j) \mathbb{1}_{\left\{\left|\sum_{k=1}^{i-1} \tilde{\Phi}(i, k)\right| \geq \frac{\varepsilon \theta_{n} n}{2}\right\}} \mathbb{1}_{\left.\left\{|\tilde{\Phi}(i, j)|<\frac{\varepsilon \theta_{n} n}{4}\right\}\right]}\right] \\
& +\frac{4}{n^{2} \theta_{n}^{2}} \sum_{i=1}^{n}\left|\mathbb{E}\left[\sum_{1 \leq j \neq k \leq i-1} \tilde{\Phi}(i, j) \tilde{\Phi}(i, k) \mathbb{1}\left\{\left|\sum_{j=1}^{i-1} \tilde{\Phi}(i, j)\right| \geq \frac{\varepsilon \theta_{n n}}{2}\right\}\right]\right|
\end{aligned}
$$

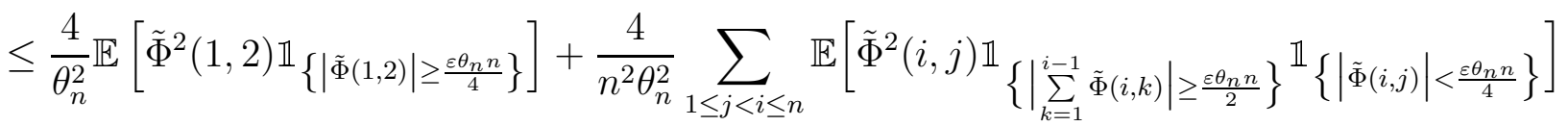

$$
\begin{aligned}
& +\frac{4}{n^{2} \theta_{n}^{2}} \sum_{i=1}^{n}\left|\mathbb{E}\left[\sum_{1 \leq j \neq k \leq i-1} \tilde{\Phi}(i, j) \tilde{\Phi}(i, k) \mathbb{1}_{\left\{\left|\sum_{j=1}^{i-1} \tilde{\Phi}(i, j)\right| \geq \frac{\varepsilon \theta_{n} n}{2}\right\}}\right]\right| \\
& =: S_{2}+S_{3}+S_{4}
\end{aligned}
$$

By (C2), we find that $S_{2} \stackrel{n \rightarrow \infty}{\longrightarrow} 0$. For $S_{3}$, we manipulate the indicators to see that

$$
\mathbb{1}_{\left\{\left|\sum_{k=1}^{i-1} \tilde{\Phi}(i, k)\right| \geq \frac{\varepsilon \theta_{n} n}{2}\right\}} \mathbb{1}_{\left\{|\tilde{\Phi}(i, j)|<\frac{\varepsilon \theta_{n} n}{4}\right\}} \leq \mathbb{1}_{\left\{\left|\sum_{\substack{1 \leq k \leq i-1 \\ k \neq j}} \tilde{\Phi}(i, k)\right| \geq \frac{\varepsilon \theta_{n} n}{4}\right\}}
$$

and use (1.7) to obtain

$$
\begin{aligned}
& S_{3} \leq \frac{4}{n^{2} \theta_{n}^{2}} \sum_{1 \leq j<i \leq n} \mathbb{E}\left[\mathbb{E}\left[\tilde{\Phi}^{2}(i, j) \mathbb{1}_{\left\{\left|\sum_{\substack{1 \leq k \leq i-1 \\
k \neq j}} \tilde{\Phi}(i, k)\right| \geq \frac{\varepsilon \theta_{n} n}{4}\right\}} \mid\left(X_{k}\right)_{\substack{k=1, \ldots, i \\
k \neq j}},\left(Z_{i, k}\right)_{k=1, \ldots, i-1}\right]\right]
\end{aligned}
$$

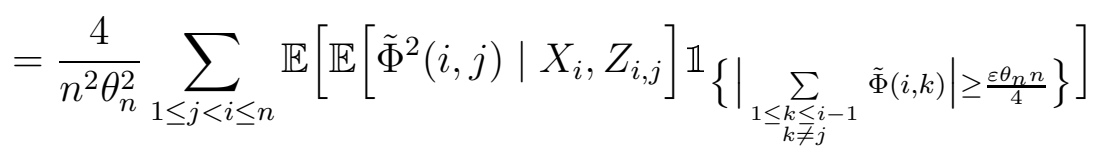

$$
\begin{aligned}
& \left.=\frac{4}{n^{2} \theta_{n}^{2}} \sum_{1 \leq j<i}^{n} \mathbb{E}\left[\tilde{G}_{j}(i, i) \mathbb{1}_{\{\mid} \sum_{\substack{1 \leq k \leq i-1 \\
k \neq j}} \tilde{\Phi}(i, k) \mid \geq \frac{\varepsilon \theta_{n} n}{4}\right\}\right]
\end{aligned}
$$

By adding another indicator, for any $\tilde{\varepsilon}>0$, this can be rewritten as

$$
\begin{aligned}
S_{3} \leq & \left.\frac{4}{n^{2} \theta_{n}^{2}} \sum_{1 \leq j<i \leq n} \mathbb{E}\left[\tilde{G}_{j}(i, i) \mathbb{1}_{\{\mid} \sum_{\substack{1 \leq k \leq i-1 \\
k \neq j}} \tilde{\Phi}(i, k) \mid \geq \frac{\varepsilon \theta_{n} n}{4}\right\}^{\mathbb{1}}\left\{|\tilde{H}(i, i)| \geq \frac{\tilde{\varepsilon} \theta_{n}^{2} n}{16 p}\right\}\right] \\
& +\frac{4}{n^{2} \theta_{n}^{2}} \sum_{1 \leq j<i \leq n} \mathbb{E}\left[\tilde{G}_{j}(i, i) \mathbb{1}_{\left.\left\{\left|\sum_{\substack{1 \leq k \leq i-1 \\
k \neq j}} \tilde{\Phi}(i, k)\right| \geq \frac{\varepsilon \theta_{n} n}{4}\right\}^{1}\left\{|\tilde{H}(i, i)|<\frac{\tilde{\varepsilon} \theta_{n}^{2} n}{16 p}\right\}\right]}\right.
\end{aligned}
$$




$$
\begin{aligned}
& \leq \frac{4}{n^{2} \theta_{n}^{2}} \sum_{1 \leq j<i \leq n} \mathbb{E}\left[\tilde{G}_{j}(i, i) \mathbb{1}_{\left\{|\tilde{H}(i, i)| \geq \frac{\tilde{\varepsilon} \theta_{n}^{2} n}{16 p}\right\}}\right]
\end{aligned}
$$

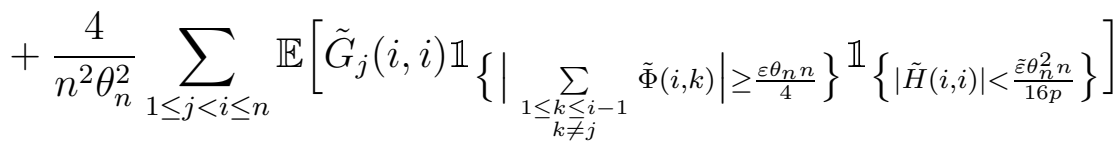

$$
\begin{aligned}
& =: S_{31}+S_{32} \text {. }
\end{aligned}
$$

For $S_{31}$ we have by independence, (1.7) and (C3)

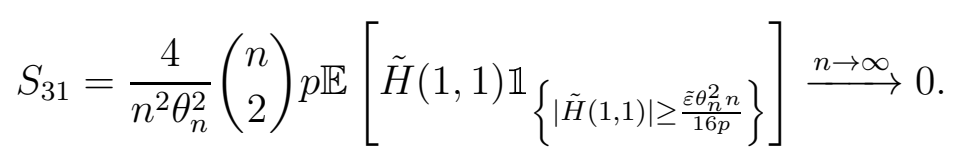

On the other hand, by applying the two indicators

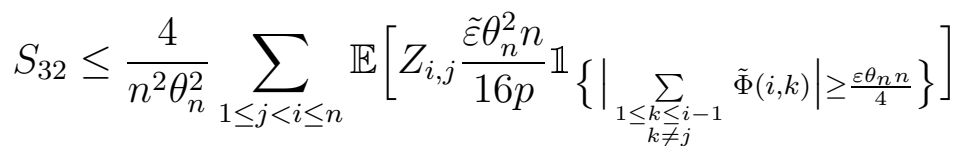

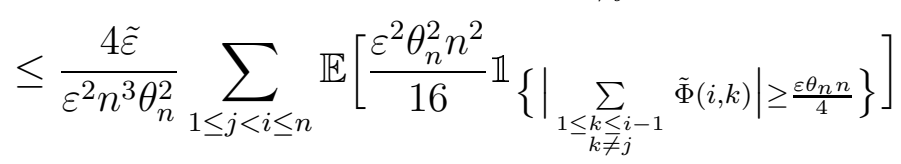

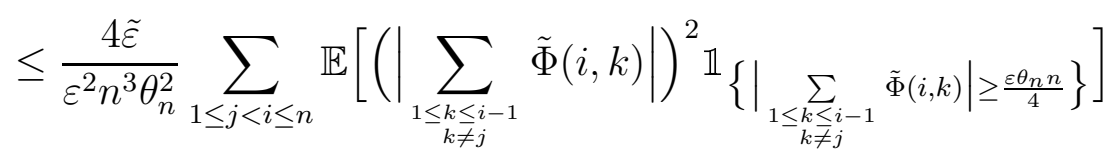

$$
\begin{aligned}
& \leq \frac{4 \tilde{\varepsilon}}{\varepsilon^{2} n^{3} \theta_{n}^{2}} \sum_{1 \leq j<i \leq n} \mathbb{E}\left[\sum_{\substack{1 \leq k \leq i-1 \\
k \neq j}} \tilde{\Phi}^{2}(i, k)+\sum_{\substack{1 \leq k \leq i-1 \\
k \neq j}} \sum_{\substack{1 \leq l \leq i-1 \\
l \neq j, k}} \tilde{\Phi}(i, k) \tilde{\Phi}(i, l)\right] \\
& \leq \frac{4 \tilde{\varepsilon}}{\varepsilon^{2} n^{3} \theta_{n}^{2}} n(n-1)\left((n-1) \mathbb{E}\left[\tilde{\Phi}^{2}(1,2)\right]+(n-1)^{2} \mathbb{E}[\tilde{\Phi}(1,2) \tilde{\Phi}(1,3)]\right)
\end{aligned}
$$

Applying Lemma 1.1, the first expectation is smaller than $\beta_{n}^{2}$. By Lemma A.2, the second expectation is 0 . Thus

$$
S_{32} \leq \frac{4(n-1)^{2} \tilde{\varepsilon}}{\varepsilon^{2} n^{2} \theta_{n}^{2}} \beta_{n}^{2} \leq \frac{8 \tilde{\varepsilon}}{\varepsilon^{2}} \frac{(n-1)^{2}}{n^{2}}
$$

by (1.3). As we may chose $\tilde{\varepsilon}>0$ arbitrarily, this shows that $S_{32} \rightarrow 0$, and together with (4.2) we obtain that $S_{3} \stackrel{n \rightarrow \infty}{\longrightarrow} 0$.

Finally, for $S_{4}$, we compute

$$
\begin{aligned}
S_{4} \leq & \frac{4}{n^{2} \theta_{n}^{2}} \sum_{i=1}^{n} \mid \mathbb{E}\left[\mathbb{1}_{\left\{|\tilde{H}(i, i)| \geq \frac{\tilde{\varepsilon} \theta_{n}^{2} n}{2 p}\right\}} \sum_{\substack{1 \leq j, k \leq i-1 \\
j \neq k}}^{i-1} \tilde{\Phi}(i, j) \tilde{\Phi}(i, k) \mathbb{1}_{\left.\left\{\left|\sum_{j=1}^{i-1} \tilde{\Phi}(i, j)\right| \geq \frac{\varepsilon \theta_{n} n}{2}\right\}\right] \mid}\right] \\
& \quad+\frac{4}{n^{2} \theta_{n}^{2}} \sum_{i=1}^{n}\left|\mathbb{E}\left[\mathbb{1}_{\left\{|\tilde{H}(i, i)|<\frac{\varepsilon \theta_{n}^{2} n}{2 p}\right\}} \sum_{\substack{1 \leq j, k \leq i-1 \\
j \neq k}} \tilde{\Phi}(i, j) \tilde{\Phi}(i, k) \mathbb{1}_{\left\{\left|\sum_{j=1}^{i-1} \tilde{\Phi}(i, j)\right| \geq \frac{\varepsilon \theta_{n} n}{2}\right\}}\right]\right| \\
= & : S_{41}+S_{42}
\end{aligned}
$$


Consider $S_{41}$ first. Because of $\left|\sum_{j \neq k} x_{j} x_{k}\right| \leq\left(\sum_{j=1}^{n} x_{j}\right)^{2}+\sum_{j=1}^{n} x_{j}^{2}$, we obtain

$$
\begin{aligned}
S_{41} \leq & \frac{4}{n^{2} \theta_{n}^{2}} \sum_{i=1}^{n} \mathbb{E}\left[\mathbb{1}_{\left\{|\tilde{H}(i, i)| \geq \frac{\tilde{\varepsilon} \theta_{n}^{2} n}{2 p}\right\}}\left(\sum_{j=1}^{i-1} \tilde{\Phi}(i, j)\right)^{2} \mathbb{1}_{\left.\left\{\left|\sum_{j=1}^{i-1} \tilde{\Phi}(i, j)\right| \geq \frac{\varepsilon \theta_{n} n}{2}\right\}\right]}\right. \\
& \quad+\frac{4}{n^{2} \theta_{n}^{2}} \sum_{i=1}^{n} \mathbb{E}\left[\mathbb{1}_{\left\{|\tilde{H}(i, i)| \geq \frac{\tilde{\theta} \theta_{n}^{2} n}{2 p}\right\}} \sum_{j=1}^{i-1} \tilde{\Phi}^{2}(i, j) \mathbb{1}\left\{\left|\sum_{j=1}^{i-1} \tilde{\Phi}(i, j)\right| \geq \frac{\varepsilon \theta_{n} n}{2}\right\}\right] \\
\leq & \frac{8}{n^{2} \theta_{n}^{2}} \sum_{i=1}^{n} \mathbb{E}\left[\mathbb{1}_{\left\{|\tilde{H}(i, i)| \geq \frac{\tilde{\varepsilon} \theta_{n}^{2} n}{2 p}\right\}} \sum_{j=1}^{i-1} \tilde{\Phi}^{2}(i, j)\right]+\frac{4}{n^{2} \theta_{n}^{2}} \sum_{i=1}^{n} \mathbb{E}\left[\mathbb{1}_{\left\{|\tilde{H}(i, i)| \geq \frac{\tilde{\varepsilon} \theta_{n}^{2} n}{2 p}\right\}} \sum_{\substack{1 \leq j, k \leq i-1 \\
j \neq k}} \tilde{\Phi}(i, j) \tilde{\Phi}(i, k)\right] \\
= & : S_{41}^{\prime}+S_{41}^{\prime \prime}
\end{aligned}
$$

For the first of these summands we obtain

$$
\begin{aligned}
S_{41}^{\prime} & =\frac{8}{n^{2} \theta_{n}^{2}} \sum_{1 \leq j<i \leq n} \mathbb{E}\left[\mathbb{1}_{\left\{|\tilde{H}(i, i)| \geq \frac{\tilde{\varepsilon} \theta_{n}^{2} n}{2 p}\right\}} \tilde{\Phi}^{2}(i, j)\right]=\frac{8}{n^{2} \theta_{n}^{2}} \sum_{1 \leq j<i \leq n} \mathbb{E}\left[\mathbb{1}_{\left\{|\tilde{H}(i, i)| \geq \frac{\tilde{\varepsilon} \theta_{n}^{2} n}{2 p}\right\}} \mathbb{E}\left[\tilde{\Phi}^{2}(i, j) \mid X_{i}, Z_{i j}\right]\right] \\
& =\frac{8}{n^{2} \theta_{n}^{2}} \sum_{1 \leq j<i \leq n} \mathbb{E}\left[\mathbb{1}_{\left\{|\tilde{H}(i, i)| \geq \frac{\tilde{\varepsilon} \theta_{n}^{2} n}{2 p}\right\}} \tilde{G}_{j}(i, i)\right]=\frac{8}{n^{2} \theta_{n}^{2}}\left(\begin{array}{c}
n \\
2
\end{array}\right) \mathbb{E}\left[\mathbb{1}_{\left\{|\tilde{H}(1,1)| \geq \frac{\tilde{\varepsilon} \theta_{n}^{2} n}{2 p}\right\}} \tilde{H}(1,1) Z_{1,2}\right] \\
& \leq \frac{4}{\theta_{n}^{2}} p \mathbb{E}\left[\mathbb{1}_{\left\{|\tilde{H}(1,1)| \geq \frac{\tilde{\varepsilon} \theta_{n}^{2} n}{2 p}\right\}} \tilde{H}(1,1)\right]
\end{aligned}
$$

which leads to $S_{41}^{\prime} \stackrel{n \rightarrow \infty}{\longrightarrow} 0$ due to (C3).

For the second summand $S_{41}^{\prime \prime}$ we estimate

$$
\begin{aligned}
S_{41}^{\prime \prime} & =\frac{4}{n^{2} \theta_{n}^{2}} \sum_{i=1}^{n} \sum_{\substack{1 \leq j, k \leq i-1 \\
j \neq k}} \mathbb{E}\left[\mathbb{E}\left[\mathbb{1}_{\left\{|\tilde{H}(i, i)| \geq \frac{\tilde{\varepsilon} \theta_{n}^{2} n}{2 p}\right\}} \tilde{\Phi}(i, j) \tilde{\Phi}(i, k) \mid X_{i}, X_{j}, Z_{i, j}, Z_{i, k}\right]\right] \\
& =\frac{4}{n^{2} \theta_{n}^{2}} \sum_{i=1}^{n} \sum_{\substack{1 \leq j, k \leq i-1 \\
j \neq k}} \mathbb{E}\left[\mathbb{1}_{\left\{|\tilde{H}(i, i)| \geq \frac{\tilde{\varepsilon} \theta_{n}^{2} n}{2 p}\right\}} \tilde{\Phi}(i, j) Z_{i, k} \mathbb{E}\left[\tilde{h}(i, k) \mid X_{i}, X_{j}, Z_{i, j}, Z_{i, k}\right]\right] \\
& =\frac{4}{n^{2} \theta_{n}^{2}} \sum_{i=1}^{n} \sum_{\substack{1 \leq j, k \leq i-1 \\
j \neq k}} \mathbb{E}\left[\mathbb{1}_{\left\{|\tilde{H}(i, i)| \geq \frac{\tilde{\varepsilon} \theta_{n}^{2} n}{2 p}\right\}} \tilde{\Phi}(i, j) Z_{i, k} \mathbb{E}\left[\tilde{h}(i, k) \mid X_{i}\right]\right]=0,
\end{aligned}
$$

which follows from (1.4). Altogether this gives $S_{41} \stackrel{n \rightarrow \infty}{\longrightarrow} 0$.

Considering now $S_{42}$ we see that (using Cauchy-Schwarz for the second inequality)

$$
\begin{aligned}
S_{42} & \leq \frac{4}{n^{2} \theta_{n}^{2}} \sum_{i=1}^{n} \mathbb{E}\left[\left|\mathbb{1}_{\left\{|\tilde{H}(i, i)|<\frac{\tilde{\varepsilon} \theta_{n}^{2} n}{2 p}\right\}} \sum_{\substack{1 \leq j, k \leq i-1 \\
j \neq k}} \tilde{\Phi}(i, j) \tilde{\Phi}(i, k) \mathbb{1}_{\left\{\left|\sum_{j=1}^{i-1} \tilde{\Phi}(i, j)\right| \geq \frac{\varepsilon \theta_{n} n}{2}\right\}}\right|\right] \\
& \leq \frac{4}{n^{2} \theta_{n}^{2}} \sum_{i=1}^{n} \mathbb{E}\left[\mathbb{1}_{\left\{|\tilde{H}(i, i)|<\frac{\varepsilon \theta_{n}^{2} n}{2 p}\right\}}\left(\sum_{j, k=1}^{i-1} \mathbb{1}_{\{j \neq k\}} \tilde{\Phi}(i, j) \tilde{\Phi}(i, k)\right)^{2}\right]^{\frac{1}{2}} \mathbb{P}\left(\left|\sum_{l=1}^{i-1} \tilde{\Phi}(i, l)\right| \geq \frac{\varepsilon \theta_{n} n}{2}\right)^{\frac{1}{2}} \\
& =: \frac{4}{n^{2} \theta_{n}^{2}} \sum_{i=1}^{n} A_{i} B_{i} .
\end{aligned}
$$


We estimate $A_{i}$ by

$$
\begin{aligned}
A_{i}^{2}= & \sum_{j, k=1}^{i-1} \mathbb{1}_{\{j \neq k\}} \mathbb{E}\left[\mathbb{1}_{\left\{|\tilde{H}(i, i)|<\frac{\tilde{\varepsilon} \theta_{n}^{2} n}{2 p}\right\}} \tilde{\Phi}^{2}(i, j) \tilde{\Phi}^{2}(i, k)\right] \\
& \quad+\sum_{\substack{j, k=1 \\
\{l, m\} \neq\{j, k\}}}^{i-1} \sum_{l, m=1}^{i-1} \mathbb{1}_{\{j \neq k\}} \mathbb{1}_{\{l \neq m\}} \mathbb{E}\left[\mathbb{1}_{\left\{|\tilde{H}(i, i)|<\frac{\tilde{\varepsilon} \theta \theta_{n}^{2} n}{2 p}\right\}} \tilde{\Phi}(i, j) \tilde{\Phi}(i, k) \tilde{\Phi}(i, l) \tilde{\Phi}(i, m)\right] \\
= & : A_{i 1}+A_{i 2}
\end{aligned}
$$

Using the properties of (conditional) expectation for $A_{i 1}$ we see that

$$
\begin{aligned}
& \mathbb{E}\left[\mathbb{1}_{\left\{|\tilde{H}(i, i)|<\frac{\tilde{\varepsilon} \theta_{n}^{2} n}{2 p}\right\}} \tilde{\Phi}^{2}(i, j) \tilde{\Phi}^{2}(i, k)\right] \\
& \quad=\mathbb{E}\left[\mathbb{E}\left[\mathbb{E}\left[\mathbb{1}_{\left\{|\tilde{H}(i, i)|<\frac{\tilde{\varepsilon} \theta_{n}^{2} n}{2 p}\right\}} \tilde{\Phi}^{2}(i, j) \tilde{\Phi}^{2}(i, k) \mid X_{i}, X_{j}, Z_{i, j}, Z_{i, k}\right] \mid X_{i}, Z_{i, j}, Z_{i, k}\right]\right] \\
& \quad=\mathbb{E}\left[\mathbb{1}_{\left\{|\tilde{H}(i, i)|<\frac{\tilde{\varepsilon} \theta_{n}^{2} n}{2 p}\right\}} \mathbb{E}\left[\tilde{\Phi}^{2}(i, j) \mid X_{i}, Z_{i, j}\right] \mathbb{E}\left[\tilde{\Phi}^{2}(i, k) \mid X_{i}, Z_{i, k}\right]\right]
\end{aligned}
$$

and by (1.7)

$$
\begin{aligned}
& =\mathbb{E}\left[\mathbb{1}_{\left\{|\tilde{H}(i, i)|<\frac{\tilde{\varepsilon} \theta_{n}^{2} n}{2 p}\right\}} \tilde{G}_{j}(i, i) \tilde{G}_{k}(i, i)\right]=\mathbb{E}\left[Z_{i, j}\right] \mathbb{E}\left[Z_{i, k}\right] \mathbb{E}\left[\mathbb{1}_{\left\{|\tilde{H}(i, i)|<\frac{\tilde{\varepsilon} \theta_{n}^{2} n}{2 p}\right\}} \tilde{H}(i, i) \tilde{H}(i, i)\right] \\
& <p^{2} \cdot \frac{\tilde{\varepsilon} \theta_{n}^{2} n}{2 p} \cdot \mathbb{E}[\tilde{H}(i, i)] \leq p \cdot \frac{\tilde{\varepsilon} \theta_{n}^{2} n}{2} \cdot \frac{\beta_{n}^{2}}{p}=\frac{\tilde{\varepsilon} \theta_{n}^{2} n}{2} \beta_{n}^{2}
\end{aligned}
$$

where in the last inequality we applied Corollary A.1.

On the other hand, for $A_{i 2}$, we know that at least one of the values $j, k, l, m$ is different from the others. Without loss of generality, this is $m$. Then,

$$
\begin{aligned}
& \mathbb{E}\left[\mathbb{1}_{\left\{|\tilde{H}(i, i)|<<\frac{\tilde{\varepsilon} \theta_{n}^{2} n}{2 p}\right\}} \tilde{\Phi}(i, j) \tilde{\Phi}(i, k) \tilde{\Phi}(i, l) \tilde{\Phi}(i, m)\right] \\
= & \mathbb{E}\left[\mathbb{E}\left[\mathbb{1}_{\left\{|\tilde{H}(i, i)|<\frac{\tilde{\varepsilon} \theta_{n}^{2} n}{2 p}\right\}} \tilde{\Phi}(i, j) \tilde{\Phi}(i, k) \tilde{\Phi}(i, l) \tilde{\Phi}(i, m) \mid Z_{i, j}, Z_{i, k}, Z_{i, l}, Z_{i, m}, X_{i}, X_{j}, X_{k}, X_{l}\right]\right] \\
= & \mathbb{E}\left[\mathbb{1}_{\left\{|\tilde{H}(i, i)|<\frac{\tilde{\varepsilon} \theta_{n}^{2} n}{2 p}\right\}} \tilde{\Phi}(i, j) \tilde{\Phi}(i, k) \tilde{\Phi}(i, l) Z_{i, m} \mathbb{E}\left[\tilde{h}(i, m) \mid X_{i}\right]\right]=0,
\end{aligned}
$$

due to (1.4) . Altogether, $A_{i}^{2}=A_{i 1} \leq n^{2} \cdot \frac{\tilde{\varepsilon} \theta_{n}^{2} n}{2} \cdot \beta_{n}^{2} \leq n^{2} \cdot \tilde{\varepsilon} n \theta_{n}^{2} \cdot \beta_{n}^{2}$, hence

$$
A_{i} \leq n \beta_{n} \theta_{n} \sqrt{\tilde{\varepsilon} n},
$$

To give a bound for $B_{i}$, we use the fact that

$\mathbb{E}\left[\left(\sum_{l=1}^{i-1} \tilde{\Phi}(i, l)\right)^{2}\right]=\mathbb{E}\left[\sum_{l=1}^{i-1} \tilde{\Phi}^{2}(i, l)\right]+\mathbb{E}\left[\sum_{l, m=1, l \neq m}^{i-1} \tilde{\Phi}(i, l) \tilde{\Phi}(i, m)\right]=\sum_{l=1}^{i-1} \mathbb{E}\left[\tilde{\Phi}^{2}(i, l)\right] \leq n \beta_{n}^{2}$,

by Lemma A.2 and Lemma 1.1, By Markov's inequality

$$
B_{i}=\mathbb{P}\left(\left|\sum_{l=1}^{i-1} \tilde{\Phi}(i, l)\right| \geq \frac{\varepsilon \theta_{n} n}{2}\right)^{1 / 2} \leq\left(\frac{4}{\varepsilon^{2} \theta_{n}^{2} n^{2}} \mathbb{E}\left[\left(\sum_{l=1}^{i-1} \tilde{\Phi}(i, l)\right)^{2}\right]\right)^{1 / 2} \leq \frac{2 \beta_{n}}{\varepsilon \theta_{n} \sqrt{n}} .
$$


Then (4.3), (4.4) and (4.5) give

$S_{42} \leq \frac{4}{n^{2} \theta_{n}^{2}} \sum_{i=1}^{n} A_{i} B_{i} \leq \frac{4}{n^{2} \theta_{n}^{2}} \sum_{i=1}^{n}\left(n \beta_{n} \theta_{n} \sqrt{\tilde{\varepsilon} n} \cdot \frac{2 \beta_{n}}{\varepsilon \theta_{n} \sqrt{n}}\right) \leq \frac{4}{n^{2} \theta_{n}^{2}} n\left(n \frac{2 \sqrt{\tilde{\varepsilon}}}{\varepsilon} \beta_{n}^{2}\right) \leq \frac{16 \sqrt{\tilde{\varepsilon}}}{\varepsilon}$

As $\tilde{\varepsilon}$ was chosen arbitrarily, we obtain, $S_{42} \stackrel{n \rightarrow \infty}{\longrightarrow} 0$, and hence $S_{4} \stackrel{n \rightarrow \infty}{\longrightarrow} 0$.

Wrapping things up, this tells us that

$$
\mathbb{E}\left[\eta_{1}\right] \leq S_{1}+T_{1} \leq S_{1}+S_{2}+S_{3}+S_{4} \stackrel{n \rightarrow \infty}{\longrightarrow} 0
$$

and thus, (B1) holds.

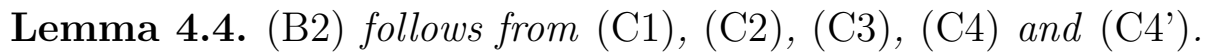

Proof. We start by calculating $\xi_{i}^{2}$ :

$$
\begin{aligned}
\xi_{i}^{2}= & \left(\xi_{i}^{(1)}+\xi_{i}^{(2)}\right)^{2}=\left(\frac{1}{n \theta_{n}} \sum_{\substack{j=1 \\
j \neq i}}^{n} \Psi_{j}(i)+\frac{1}{n \theta_{n}} \sum_{j=1}^{i-1} \tilde{\Phi}(i, j)\right)^{2} \\
= & \frac{1}{n^{2} \theta_{n}^{2}} \sum_{\substack{j=1 \\
j \neq i}}^{n} \Psi_{j}^{2}(i)+\frac{1}{n^{2} \theta_{n}^{2}} \sum_{\substack{j, k=1 \\
j, k \neq i, j \neq k}}^{n} \Psi_{j}(i) \Psi_{k}(i)+2 \frac{1}{n^{2} \theta_{n}^{2}} \sum_{\substack{j=1 \\
j \neq i}}^{n} \sum_{k=1}^{i-1} \Psi_{j}(i) \tilde{\Phi}(i, k) \\
& +\sum_{j=1}^{i-1} \tilde{\Phi}^{2}(i, j)+\frac{1}{n^{2} \theta_{n}^{2}} \sum_{\substack{j, k=1 \\
j \neq k}}^{i-1} \tilde{\Phi}(i, j) \tilde{\Phi}(i, k)
\end{aligned}
$$

We will compute the sum (in $i$ ) of the conditional expectations for of each of these summands. Let us start with the second one: First observe that for any choice of $i \neq j, k$ we have $\Psi_{k}(i)=\Psi_{j}(i) Z_{i k} / Z_{i j}$. Hence, using that $Z_{i j}^{2}=Z_{i j}$ we get

$$
\begin{aligned}
& \sum_{i=1}^{n} \frac{1}{n^{2} \theta_{n}^{2}} \sum_{\substack{j, k=1 \\
j, k \neq i, j \neq k}}^{n} \mathbb{E}\left[\Psi_{j}(i) \Psi_{k}(i) \mid\left(X_{k}\right)_{k=1, \ldots, i-1},\left(Z_{l, m}\right)_{\substack{l=1, \ldots, i-1, m=1, \ldots, n, m \neq l}}\right] \\
& =\sum_{i=1}^{n} \frac{1}{n^{2} \theta_{n}^{2}} \sum_{\substack{j, k=1 \\
j, k \neq i, j \neq k}}^{n} \mathbb{E}\left[Z_{i, k} \Psi_{j}^{2}(i) \mid\left(X_{k}\right)_{k=1, \ldots, i-1},\left(Z_{l, m}\right)_{\substack{l=1, \ldots, i-1, m=1, \ldots, n, m \neq l}}\right] \\
& =\sum_{i=1}^{n} \frac{1}{n^{2} \theta_{n}^{2}} \sum_{\substack{j=1 \\
j \neq i}}^{n}\left(\sum_{\substack{k=1 \\
k \neq j}}^{i-1} Z_{i, k}+\sum_{\substack{k=i+1 \\
k \neq i, j}}^{n} p\right) \mathbb{E}\left[\Psi_{j}^{2}(i) \mid\left(X_{k}\right)_{k=1, \ldots, i-1},\left(Z_{l, m}\right)_{\substack{l=1, \ldots, i-1, m=1, \ldots, n, m \neq l}}\right]
\end{aligned}
$$

where we applied measurability of $Z_{i, k}$ for $k<i$ with respect to the condition and independence of the condition for $Z_{i, k}, k>i$. 
The conditional expectation of first two summands in (4.6) together then is

$$
\begin{aligned}
\eta_{21} & :=\sum_{i=1}^{n} \frac{1}{n^{2} \theta_{n}^{2}} \sum_{\substack{j=1 \\
j \neq i}}^{n} \mathbb{E}\left[\Psi_{j}^{2}(i) \mid\left(X_{k}\right)_{k=1, \ldots, i-1},\left(Z_{l, m}\right)_{l=1, \ldots, i-1,}\right] \cdot\left(\sum_{\substack{k=1, \ldots, n, m \neq l \\
k \neq j}}^{i-1} Z_{i, k}+\sum_{\substack{k=i+1 \\
k \neq i, j}}^{n} p+1\right) \\
& =\sum_{i=1}^{n} \frac{1}{n^{2} \theta_{n}^{2}} \sum_{\substack{j=1 \\
j \neq i}}^{n} \mathbb{E}\left[Z_{i j}^{2} \mathbb{E}\left[h(i, j) \mid X_{i}\right]^{2} \mid\left(Z_{l, m}\right)_{\substack{l=1, \ldots, i-1, m=1, \ldots, n, m \neq l}}\right] \cdot\left(\sum_{\substack{k=1 \\
k \neq j}}^{i-1} Z_{i, k}+\sum_{\substack{k=i+1 \\
k \neq i, j}}^{n} p+1\right) \\
& =\sum_{i=1}^{n} \frac{1}{n^{2} \theta_{n}^{2}} \sum_{\substack{j=1 \\
j \neq i}}^{n} \mathbb{E}\left[Z_{i j}^{2} \mathbb{E}\left[\mathbb{E}\left[h(i, j) \mid X_{i}\right]^{2}\right] \mid\left(Z_{l, m}\right)_{\substack{l=1, \ldots, i-1, m=1, \ldots, n, m \neq l}}^{i-1} \sum_{\substack{k=1 \\
k \neq j}}^{n} Z_{i, k}+\sum_{\substack{k=i+1 \\
k \neq i, j}}^{n} p+1\right) \\
& =\frac{\gamma_{n}^{2}}{n^{2} p \theta_{n}^{2}} \sum_{i=1}^{n} \sum_{\substack{j=1 \\
j \neq i}}^{n} \mathbb{E}\left[Z_{i, j} \cdot\left(\sum_{\substack{k=1 \\
k \neq j}}^{i-1} Z_{i, k}+\sum_{\substack{k=i+1 \\
k \neq i, j}}^{n} p+1\right) \mid\left(Z_{l, i}\right)_{l=1, \ldots, i-1}\right] .
\end{aligned}
$$

since $\mathbb{E}\left[\mathbb{E}\left[h(i, j) \mid X_{i}\right]^{2}\right]$ is given by Lemma 1.1, and $Z_{i, j}^{2}=Z_{i, j}$. It is easily seen that for this term, the relation

$$
\mathbb{E}\left[\eta_{21}\right]=\frac{\gamma_{n}^{2}}{n^{2} p \theta_{n}^{2}} n(n-1)((n-2) p+1) p \sim \frac{n p \gamma_{n}^{2}}{\theta_{n}^{2}}
$$

holds. Next, the conditional expectation of the fourth summand in (4.6) can be computed as:

$$
\begin{aligned}
\eta_{22} & :=\frac{1}{n^{2} \theta_{n}^{2}} \sum_{i=1}^{n} \sum_{j=1}^{i-1} \mathbb{E}\left[\tilde{\Phi}^{2}(i, j) \mid\left(X_{k}\right)_{k=1, \ldots, i-1},\left(\begin{array}{r}
\left(Z_{l, m}\right)_{l=1, \ldots, i-1,} \\
m=1, \ldots, n, m \neq l
\end{array}\right]\right. \\
& =\frac{1}{n^{2} \theta_{n}^{2}} \sum_{i=1}^{n} \sum_{j=1}^{i-1} \mathbb{E}\left[\tilde{\Phi}^{2}(i, j) \mid X_{j}, Z_{i, j}\right]=\frac{1}{n^{2} \theta_{n}^{2}} \sum_{i=1}^{n} \sum_{j=1}^{i-1} \tilde{G}_{i}(j, j)
\end{aligned}
$$

by (1.7). By Corollary A.1 the expectation for this term satisfies

$$
\mathbb{E}\left[\eta_{22}\right]=\left(\begin{array}{l}
n \\
2
\end{array}\right) \frac{1}{n^{2} \theta_{n}^{2}}\left(\beta_{n}^{2}-2 \gamma_{n}^{2}\right) \sim \frac{\beta_{n}^{2}-2 \gamma_{n}^{2}}{2 \theta_{n}^{2}}
$$

Furthermore, the sum of the conditional expectations of fifth summand in (4.6) by (1.7) is $\eta_{23}:=\frac{1}{n^{2} \theta_{n}^{2}} \sum_{i=1}^{n} \sum_{\substack{j, k=1 \\ j \neq k}}^{i-1} \tilde{G}_{i}(j, k)$. For the third summand in (4.6) we obtain:

$$
\eta_{24}:=\frac{2}{n^{2} \theta_{n}^{2}} \sum_{i=1}^{n} \sum_{\substack{j=1 \\ j \neq i}}^{n} \sum_{m=1}^{i-1} \mathbb{E}\left[\Psi_{j}(i) \tilde{\Phi}(i, m) \mid X_{m},\left(Z_{l, i}\right)_{l=1, \ldots, i-1,}\right]
$$

Then $\eta_{2} \sim \eta_{21}+\eta_{22}+\eta_{23}+\eta_{24}$. One can immediately conclude from (4.7) and (4.8) that $\mathbb{E}\left[\eta_{21}+\eta_{22}\right] \sim \frac{n p \gamma_{n}^{2}}{\theta_{n}^{2}}+\frac{\beta_{n}^{2}-2 \gamma_{n}^{2}}{2 \theta_{n}^{2}} \sim \frac{\frac{1}{2} \beta_{n}^{2}+n p \gamma_{n}^{2}}{\theta_{n}^{2}}$ and by definition of $\theta_{n}^{2}$ (cf. (1.3) $)$

$$
\mathbb{E}\left[\eta_{21}+\eta_{22}\right] \stackrel{n \rightarrow \infty}{\longrightarrow} 1 \text {. }
$$


Let us split up $\eta_{22}$, by choosing $\tilde{\varepsilon}>0$ arbitrarily:

$$
\begin{aligned}
\eta_{22} & =\frac{1}{n^{2} \theta_{n}^{2}} \sum_{i=1}^{n} \sum_{j=1}^{i-1} \tilde{G}_{i}(j, j) \mathbb{1}_{\left\{|\tilde{H}(j, j)|<\frac{\varepsilon \theta_{n}^{2} n}{p}\right\}}+\frac{1}{n^{2} \theta_{n}^{2}} \sum_{i=1}^{n} \sum_{j=1}^{i-1} \tilde{G}_{i}(j, j) \mathbb{1}_{\left\{|\tilde{H}(j, j)| \geq \frac{\tilde{\varepsilon} \theta_{n}^{2} n}{p}\right\}} \\
& =: \eta_{22}^{\prime}+\eta_{22}^{\prime \prime}
\end{aligned}
$$

Consider the second summand first. By definition

$\mathbb{E}\left[\eta_{22}^{\prime \prime}\right]=\frac{1}{n^{2} \theta_{n}^{2}} \sum_{i=1}^{n} \sum_{j=1}^{i-1} \mathbb{E}\left[Z_{i, j} \tilde{H}(j, j) \mathbb{1}_{\left\{|\tilde{H}(j, j)| \geq \frac{\tilde{\varepsilon}_{n}^{2} n}{p}\right\}}\right]=\frac{1}{n^{2}}\left(\begin{array}{l}n \\ 2\end{array}\right) \frac{p}{\theta_{n}^{2}} \mathbb{E}\left[\tilde{H}(1,1) \mathbb{1}_{\left\{|\tilde{H}(1,1)| \geq \frac{\tilde{\varepsilon} \theta_{n}^{2} n}{p}\right\}}\right]$.

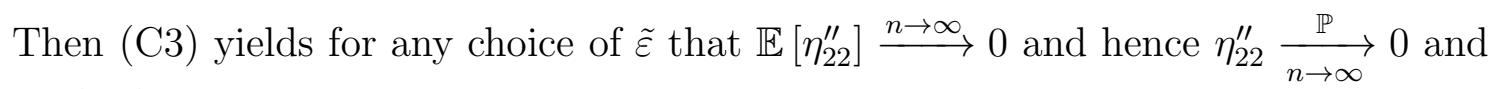
by (4.9)

$$
\mathbb{E}\left[\eta_{21}+\eta_{22}^{\prime}\right] \stackrel{n \rightarrow \infty}{\longrightarrow} 1
$$

To compute $\mathbb{E}\left[\left(\eta_{21}+\eta_{22}^{\prime}\right)^{2}\right]$ we define $\Lambda_{j}(i):=\sum_{\substack{k=1 \\ k \neq j}}^{i-1} Z_{i, k}+\sum_{\substack{k=i+1 \\ k \neq i, j}}^{n} p+1$ and compute

$\mathbb{E}\left[\eta_{21}^{2}\right]=\frac{\gamma_{n}^{4}}{n^{4} p^{2} \theta_{n}^{4}} \sum_{i, i^{\prime}=1}^{n} \sum_{\substack{j=1 \\ j \neq i}}^{n} \sum_{\substack{j^{\prime}=1 \\ j^{\prime} \neq i^{\prime}}}^{n} \mathbb{E}\left[\mathbb{E}\left[\Lambda_{j}(i) Z_{i, j} \mid\left(Z_{l, i}\right)_{l=1, \ldots, i-1}\right] \cdot \mathbb{E}\left[\Lambda_{j^{\prime}}\left(i^{\prime}\right) Z_{i^{\prime}, j^{\prime}} \mid\left(Z_{l, i^{\prime}}\right)_{l=1, \ldots, i^{\prime}-1}\right]\right]$

If $i=i^{\prime}, j=j^{\prime}$, by Jensen's inequality we find

$$
\begin{aligned}
\mathbb{E}\left[\mathbb{E}\left[\Lambda_{j}(i) Z_{i, j} \mid\left(Z_{l, i}\right)_{l=1, \ldots, i-1}\right] \cdot \mathbb{E}\left[\Lambda_{j^{\prime}}\left(i^{\prime}\right) Z_{i^{\prime}, j^{\prime}} \mid\left(Z_{l, i^{\prime}}\right)_{l=1, \ldots, i^{\prime}-1}\right]\right] \\
\leq \mathbb{E}\left[Z_{i j}^{2} \Lambda_{j}^{2}(i)\right]=\mathbb{E}\left[Z_{i j} Z_{i^{\prime} j^{\prime}} \Lambda_{j}(i) \Lambda_{j^{\prime}}\left(i^{\prime}\right)\right]
\end{aligned}
$$

If $i=i^{\prime}, j \neq j^{\prime}, \Lambda_{j}(i)$ and $\Lambda_{j^{\prime}}(i)$ are $\left(Z_{l, i}\right)_{l=1, \ldots, i-1}$-measurable. Then, dragging the second conditional into the first one and additionally conditioning on $Z_{i, j}$ in it (which is possible due to $j \neq j^{\prime}$ ),

$$
\begin{aligned}
\mathbb{E} & {\left[\mathbb{E}\left[\Lambda_{j}(i) Z_{i, j} \mid\left(Z_{l, i}\right)_{l=1, \ldots, i-1}\right] \cdot \mathbb{E}\left[\Lambda_{j^{\prime}}\left(i^{\prime}\right) Z_{i^{\prime}, j^{\prime}} \mid\left(Z_{l, i^{\prime}}\right)_{l=1, \ldots, i^{\prime}-1}\right]\right] } \\
& =\mathbb{E}\left[\Lambda_{j}(i) \Lambda_{j^{\prime}}(i) \cdot \mathbb{E}\left[Z_{i, j} \mid\left(Z_{l, i}\right)_{l=1, \ldots, i-1}\right] \mathbb{E}\left[Z_{i, j^{\prime}} \mid\left(Z_{l, i}\right)_{l=1, \ldots, i-1}\right]\right] \\
& =\mathbb{E}\left[\Lambda_{j}(i) \Lambda_{j^{\prime}}(i) \cdot \mathbb{E}\left[\mathbb{E}\left[Z_{i, j} Z_{i, j^{\prime}} \mid\left(Z_{l, i}\right)_{l=1, \ldots, i-1, j}\right] \mid\left(Z_{l, i}\right)_{l=1, \ldots, i-1}\right]\right] \\
& =\mathbb{E}\left[Z_{i, j} Z_{i, j^{\prime}} \cdot \Lambda_{j}(i) \Lambda_{j^{\prime}}(i)\right],
\end{aligned}
$$

where the last equality is due to the above measurability again and then applying law of total expectation.

Finally, if $i \neq i^{\prime}$, by independence and law of total expectation we have

$$
\begin{gathered}
\mathbb{E}\left[\mathbb{E}\left[\Lambda_{j}(i) Z_{i, j} \mid\left(Z_{l, i}\right)_{l=1, \ldots, i-1}\right] \cdot \mathbb{E}\left[\Lambda_{j^{\prime}}\left(i^{\prime}\right) Z_{i^{\prime}, j^{\prime}} \mid\left(Z_{l, i^{\prime}}\right)_{l=1, \ldots, i^{\prime}-1}\right]\right] \\
=\mathbb{E}\left[\Lambda_{j}(i) Z_{i, j}\right] \cdot \mathbb{E}\left[\Lambda_{j^{\prime}}\left(i^{\prime}\right) Z_{i^{\prime}, j^{\prime}}\right]=\mathbb{E}\left[Z_{i, j} Z_{i, j^{\prime}} \cdot \Lambda_{j}(i) \Lambda_{j^{\prime}}(i)\right] .
\end{gathered}
$$


Thus

$$
\mathbb{E}\left[\eta_{21}^{2}\right] \leq \frac{\gamma_{n}^{4}}{n^{4} p^{2} \theta_{n}^{4}} \sum_{i=1}^{n} \sum_{\substack{i^{\prime}=1 \\ i^{\prime}}}^{n} \sum_{\substack{j=1 \\ j \neq i}}^{n} \sum_{\substack{j^{\prime}=1 \\ j^{\prime} \neq i^{\prime}}}^{n} \mathbb{E}\left[Z_{i, j} Z_{i^{\prime}, j^{\prime}} \Lambda_{j}(i) \Lambda_{j^{\prime}}\left(i^{\prime}\right)\right]
$$

By Lemma A.4 this immediately leads to $\mathbb{E}\left[\eta_{21}^{2}\right] \lesssim \frac{(n p)^{2} \gamma_{n}^{4}}{\theta_{n}^{4}}$. Moreover,

$$
\begin{aligned}
\mathbb{E}\left[\eta_{21} \eta_{22}^{\prime}\right] & =\mathbb{E}\left[\frac{\gamma_{n}^{2}}{n^{2} p \theta_{n}^{2}} \sum_{i=1}^{n} \sum_{\substack{j=1 \\
j \neq i}}^{n} \mathbb{E}\left[Z_{i, j} \Lambda_{j}(i) \mid\left(Z_{l, i}\right)_{l=1, \ldots, i-1}\right] \frac{1}{n^{2} \theta_{n}^{2}} \sum_{i^{\prime}=1}^{n} \sum_{j^{\prime}=1}^{i^{\prime}-1} \tilde{G}_{i^{\prime}}\left(j^{\prime}, j^{\prime}\right) \mathbb{1}_{\left\{\left|\tilde{H}\left(j^{\prime}, j^{\prime}\right)\right|<\frac{\varepsilon \theta_{n}^{2} n}{p}\right\}}\right] \\
& \leq \mathbb{E}\left[\frac{\gamma_{n}^{2}}{n^{2} p \theta_{n}^{2}} \sum_{i=1}^{n} \sum_{\substack{j=1 \\
j \neq i}}^{n} \mathbb{E}\left[Z_{i, j} \Lambda_{j}(i) \mid\left(Z_{l, i}\right)_{l=1, \ldots, i-1}\right] \cdot \frac{1}{n^{2} \theta_{n}^{2}} \sum_{i^{\prime}=1}^{n} \sum_{j^{\prime}=1}^{i^{\prime}-1} \tilde{G}_{i^{\prime}}\left(j^{\prime}, j^{\prime}\right)\right]
\end{aligned}
$$

Similarly to the previous step we get by (1.7)

$$
\mathbb{E}\left[\eta_{21} \eta_{22}^{\prime}\right] \leq \frac{\gamma_{n}^{2}}{n^{4} p \theta_{n}^{4}} \sum_{i, i^{\prime}=1}^{n} \sum_{\substack{j=1 \\ j \neq i}}^{n} \sum_{j^{\prime}=1}^{i^{\prime}-1} \mathbb{E}\left[\Lambda_{j}(i) Z_{i, j} Z_{i^{\prime}, j^{\prime}} \tilde{h}^{2}\left(i^{\prime}, j^{\prime}\right)\right]
$$

Now the $\tilde{h}$ term only depends on the $X_{i^{\prime}}$, such that is independent of $\Lambda_{j}(i) Z_{i, j} Z_{i^{\prime}, j^{\prime}}$. By Lemma 1.1, $\mathbb{E}\left[\tilde{h}^{2}(i, j)\right]=\frac{\beta_{n}^{2}-2 \gamma_{n}^{2}}{p} \leq \frac{\beta_{n}^{2}}{p}$ such that

$$
\mathbb{E}\left[\eta_{21} \eta_{22}^{\prime}\right] \leq \frac{\gamma_{n}^{2} \beta_{n}^{2}}{n^{4} p^{2} \theta_{n}^{4}} \sum_{i=1}^{n} \sum_{\substack{j=1 \\ j \neq i}}^{n} \sum_{i^{\prime}=1}^{n} \sum_{j^{\prime}=1}^{i^{\prime}-1} \mathbb{E}\left[\Lambda_{j}(i) Z_{i, j} Z_{i^{\prime}, j^{\prime}}\right]
$$

Applying Lemma A.5 yields $\mathbb{E}\left[\eta_{21} \eta_{22}^{\prime}\right] \lesssim \frac{n p}{2 \theta_{n}^{4}} \gamma_{n}^{2} \beta_{n}^{2}$. Finally,

$$
\begin{aligned}
& \mathbb{E}\left[\left(\eta_{22}^{\prime}\right)^{2}\right]=\frac{1}{n^{4} \theta_{n}^{4}} \sum_{i=1}^{n} \sum_{j=1}^{i-1} \sum_{i^{\prime}=1}^{n} \sum_{j^{\prime}=1}^{i^{\prime}-1} \mathbb{E}\left[\tilde{G}_{i}(j, j) \mathbb{1}_{\left\{|\tilde{H}(j, j)|<\frac{\tilde{\varepsilon} \theta_{n}^{2} n}{p}\right\}} \tilde{G}_{i^{\prime}}\left(j^{\prime}, j^{\prime}\right) \mathbb{1}_{\left\{\left|\tilde{H}\left(j^{\prime}, j^{\prime}\right)\right|<\frac{\tilde{\varepsilon} \theta_{n}^{2} n}{p}\right\}}\right] \\
& =\frac{1}{n^{4} \theta_{n}^{4}} \sum_{i=1}^{n} \sum_{j=1}^{i-1} \sum_{i^{\prime}=1}^{n} \mathbb{E}\left[\tilde{G}_{i}(j, j) \mathbb{1}_{\left\{|\tilde{H}(j, j)|<\frac{\tilde{\varepsilon} \theta_{n}^{2} n}{p}\right\}} \tilde{G}_{i^{\prime}}(j, j) \mathbb{1}_{\left\{|\tilde{H}(j, j)|<\frac{\tilde{\varepsilon} \theta_{n}^{2} n}{p}\right\}}\right] \\
& +\frac{1}{n^{4} \theta_{n}^{4}} \sum_{i=1}^{n} \sum_{j=1}^{i-1} \sum_{i^{\prime}=1}^{n} \sum_{\substack{j^{\prime}=1 \\
j^{\prime} \neq j}}^{i^{\prime}-1} \mathbb{E}\left[\tilde{G}_{i}(j, j) \mathbb{1}_{\left\{|\tilde{H}(j, j)|<\frac{\tilde{\varepsilon} \theta_{n}^{2} n}{p}\right\}} \tilde{G}_{i^{\prime}}\left(j^{\prime}, j^{\prime}\right) \mathbb{1}_{\left\{\left|\tilde{H}\left(j^{\prime}, j^{\prime}\right)\right|<\frac{\tilde{\varepsilon} \theta_{n}^{2} n}{p}\right\}}\right]
\end{aligned}
$$

and applying (1.7) to both sums gives

$$
\begin{aligned}
& \leq \frac{1}{n^{4} \theta_{n}^{4}} \sum_{i=1}^{n} \sum_{j=1}^{i-1} \sum_{i^{\prime}=1}^{n} \mathbb{E}\left[Z_{i, j} Z_{i^{\prime}, j} \tilde{H}^{2}(j, j) \mathbb{1}_{\left\{|\tilde{H}(j, j)|<\frac{\tilde{\varepsilon} \theta_{n}^{2} n}{p}\right\}}\right] \\
& +\frac{1}{n^{4} \theta_{n}^{4}} \sum_{i=1}^{n} \sum_{j=1}^{i-1} \sum_{\substack{i^{\prime}=1 \\
i^{\prime}=1 \\
j^{\prime} \neq j}} \sum_{\substack{j^{\prime}-1 \\
j^{\prime} \neq j}}\left[Z_{i, j} Z_{i^{\prime}, j^{\prime}} \tilde{H}(j, j) \tilde{H}\left(j^{\prime}, j^{\prime}\right)\right]
\end{aligned}
$$


By independence we arrive at

$$
\begin{aligned}
& \mathbb{E}\left[\left(\eta_{22}^{\prime}\right)^{2}\right] \leq \frac{1}{n^{4} \theta_{n}^{4}} \sum_{i=1}^{n} \sum_{j=1}^{i-1} \sum_{i^{\prime}=1}^{n} \mathbb{E}\left[Z_{i, j} Z_{i^{\prime}, j}\right] \mathbb{E}\left[\tilde{H}^{2}(j, j) \mathbb{1}_{\left\{|\tilde{H}(j, j)|<\frac{\tilde{\varepsilon} \theta n_{n}^{2}}{p}\right\}}\right] \\
& +\frac{1}{n^{4} \theta_{n}^{4}} \sum_{i=1}^{n} \sum_{j=1}^{i-1} \sum_{i^{\prime}=1}^{n} \sum_{\substack{i^{\prime}=1 \\
j^{\prime} \neq j}} \mathbb{E}\left[Z_{i, j}\right] \mathbb{E}\left[Z_{i^{\prime}, j^{\prime}}\right] \mathbb{E}[\tilde{H}(j, j)] \mathbb{E}\left[\tilde{H}\left(j^{\prime}, j^{\prime}\right)\right] \\
& \leq \frac{1}{n^{4} \theta_{n}^{4}} \sum_{i=1}^{n} \sum_{j=1}^{i-1} \sum_{i^{\prime}=1}^{n} \mathbb{E}\left[Z_{i, j} Z_{i^{\prime}, j}\right] \frac{\tilde{\varepsilon} \theta_{n}^{2} n}{p} \mathbb{E}[\tilde{H}(j, j)] \\
& +\frac{1}{n^{4} \theta_{n}^{4}} \sum_{i=1}^{n} \sum_{j=1}^{i-1} \sum_{i^{\prime}=1}^{n} \sum_{j^{\prime}=1}^{i^{\prime}-1} \mathbb{E}_{j^{\prime} \neq j}\left[Z_{i, j}\right] \mathbb{E}\left[Z_{i^{\prime}, j^{\prime}}\right] \mathbb{E}[\tilde{H}(j, j)] \mathbb{E}\left[\tilde{H}\left(j^{\prime}, j^{\prime}\right)\right] \\
& \leq \frac{1}{n^{4} \theta_{n}^{4}} \sum_{i=1}^{n} \sum_{j=1}^{i-1} \sum_{i^{\prime}=1}^{n} \mathbb{E}\left[Z_{i, j} Z_{i^{\prime}, j}\right] \frac{\tilde{\varepsilon} \theta_{n}^{2} n}{p} \frac{\beta_{n}^{2}}{p}+\frac{1}{n^{4} \theta_{n}^{4}} \sum_{i=1}^{n} \sum_{j=1}^{i-1} \sum_{i^{\prime}=1}^{n} \sum_{\substack{j^{\prime}=1 \\
i^{\prime}-1}} \mathbb{E}\left[Z_{i, j}\right] \mathbb{E}\left[Z_{i^{\prime}, j^{\prime}}\right]\left(\frac{\beta_{n}^{2}}{p}\right)^{2}
\end{aligned}
$$

where we applied Corollary A.1 and used the bound $\beta_{n}^{2}-2 \gamma_{n}^{2} \leq \beta_{n}^{2}$. By $Z_{i, j}^{2}=Z_{i, j}$

$$
\begin{aligned}
\mathbb{E}\left[\left(\eta_{22}^{\prime}\right)^{2}\right] & \leq \frac{1}{n^{4} \theta_{n}^{4}} \frac{\tilde{\varepsilon} \theta_{n}^{2} n}{p} \frac{\beta_{n}^{2}}{p}\left(\left(\begin{array}{l}
n \\
2
\end{array}\right) p+\left(\begin{array}{l}
n \\
2
\end{array}\right)(n-1) p^{2}\right)+\frac{1}{n^{4} \theta_{n}^{4}}\left(\begin{array}{l}
n \\
2
\end{array}\right)^{2} p^{2}\left(\frac{\beta_{n}^{2}}{p}\right)^{2} \\
& \sim \frac{1}{n^{4} \theta_{n}^{4}} \frac{\tilde{\varepsilon} \theta_{n}^{2} n}{p} \frac{\beta_{n}^{2}}{p}\left(\begin{array}{l}
n \\
2
\end{array}\right)(n-1) p^{2}+\frac{1}{n^{4} \theta_{n}^{4}}\left(\begin{array}{l}
n \\
2
\end{array}\right)^{2} p^{2}\left(\frac{\beta_{n}^{2}}{p}\right)^{2} \sim \tilde{\varepsilon}+\frac{\beta_{n}^{4}}{4 \theta_{n}^{4}}
\end{aligned}
$$

by $\frac{\beta_{n}^{2}}{2} \leq \theta_{n}^{2}$. Thus, after a quick calculation

$\mathbb{E}\left[\left(\eta_{21}+\eta_{22}^{\prime}\right)^{2}\right] \lesssim \frac{(n p)^{2} \gamma_{n}^{4}}{\theta_{n}^{4}}+2 \frac{n p}{2 \theta_{n}^{4}} \gamma_{n}^{2} \beta_{n}^{2}+\frac{\beta_{n}^{4}}{4 \theta_{n}^{4}}+\tilde{\varepsilon}=\frac{1}{\theta_{n}^{4}}\left(n p \gamma_{n}^{2}+\frac{1}{2} \beta_{n}^{2}\right)^{2}+\tilde{\varepsilon}=1+\tilde{\varepsilon}$

Putting this together with (4.10), we obtain $\mathbb{V}\left(\eta_{21}+\eta_{22}^{\prime}\right) \lesssim \tilde{\varepsilon}+o(1)$.

Hence $\eta_{21}+\eta_{22}^{\prime}$ converges in probability to the limit of its expectation, which is 1 . It remains to show that $\eta_{23}, \eta_{24} \underset{n \rightarrow \infty}{\stackrel{\mathbb{P}}{\longrightarrow}} 0$, then $\eta_{21}+\eta_{22}+\eta_{23}+\eta_{24} \underset{n \rightarrow \infty}{\stackrel{\mathbb{P}}{\longrightarrow}} 1$. We start with $\eta_{23}$. By similar calculations as above:

$$
\begin{aligned}
& \mathbb{E}\left[\eta_{23}^{2}\right]=\frac{1}{n^{4} \theta_{n}^{4}} \sum_{i=1}^{n} \sum_{\substack{j, k=1 \\
j \neq k}}^{i-1} \sum_{i^{\prime}=1}^{n} \sum_{\substack{j^{\prime}, k^{\prime}=1 \\
j^{\prime} \neq k^{\prime}}}^{i^{\prime}-1} \mathbb{E}\left[\tilde{G}_{i}(j, k) \tilde{G}_{i^{\prime}}\left(j^{\prime}, k^{\prime}\right)\right] \\
& =\frac{1}{n^{4} \theta_{n}^{4}} \sum_{i=1}^{n} \sum_{\substack{j, k=1 \\
j \neq k}}^{i-1} \sum_{i^{\prime}=1}^{n} \sum_{\substack{j^{\prime}, k^{\prime}=1 \\
j^{\prime} \neq k^{\prime}}}^{i^{\prime}-1} \mathbb{E}\left[\tilde{\Phi}\left(i^{\prime}, j^{\prime}\right) \tilde{\Phi}\left(i^{\prime}, k^{\prime}\right) \tilde{\Phi}(i, j) \tilde{\Phi}(i, k)\right]
\end{aligned}
$$

which converges to 0 by Lemma A.6. Thus $\eta_{23}^{2} \underset{n \rightarrow \infty}{\stackrel{\mathbb{P}}{\longrightarrow}} 0$ follows. 
Finally for $\eta_{24}$,

$$
\begin{array}{r}
\mathbb{E}\left[\eta_{24}^{2}\right]=\frac{4}{n^{4} \theta_{n}^{4}} \sum_{i=1}^{n} \sum_{\substack{j=1 \\
j \neq i}}^{n} \sum_{m=1}^{i-1} \sum_{\substack { i^{\prime}=1 \\
\begin{subarray}{c}{j^{\prime}=1 \\
j^{\prime} \neq i^{\prime}{ i ^ { \prime } = 1 \\
\begin{subarray} { c } { j ^ { \prime } = 1 \\
j ^ { \prime } \neq i ^ { \prime } } }\end{subarray}}^{n} \sum_{m^{\prime}=1}^{i^{\prime}-1} \mathbb{E}\left[\mathbb{E}\left[\Psi_{j}(i) \tilde{\Phi}(i, m) \mid X_{m},\left(Z_{l, i}\right)_{l=1, \ldots, i-1}\right]\right. \\
\left.\cdot \mathbb{E}\left[\Psi_{j^{\prime}}\left(i^{\prime}\right) \tilde{\Phi}\left(i^{\prime}, m^{\prime}\right) \mid X_{m^{\prime}},\left(Z_{l, i^{\prime}}\right)_{l=1, \ldots, i^{\prime}-1}\right]\right]
\end{array}
$$

which converges to 0 by Lemma A.7 and consequently $\eta_{24} \underset{n \rightarrow \infty}{\stackrel{P}{\longrightarrow}} 0$. By the above estimates for the $\eta_{2 i}, i=1, \ldots 4$ we conclude

$$
\eta_{2} \underset{n \rightarrow \infty}{\stackrel{\mathbb{P}}{\longrightarrow}} 1
$$

which completes the proof.

\section{Alternative Conditions for the Central Limit Theorem}

As mentioned above, it may be sometimes cumbersome to check the condition (C1) (C3) in Theorem 1.5. We now give an alternative.

Proposition 5.1. The conditions (C1) $-(\mathrm{C} 3)$ follow from

$$
\begin{array}{r}
n^{2} \theta_{n}^{-2} \mathbb{E}\left[\Psi_{2}^{2}(1) \mathbb{1}_{\left\{\left|\Psi_{2}(1)\right| \geq \varepsilon \theta_{n}\right\}}\right] \stackrel{n \rightarrow \infty}{\longrightarrow} 0 \\
\theta_{n}^{-2} \mathbb{E}\left[\Phi^{2}(1,2) \mathbb{1}_{\left\{|\Phi(1,2)| \geq \varepsilon \theta_{n} n\right\}}\right] \stackrel{n \rightarrow \infty}{\longrightarrow} 0 \\
p \theta_{n}^{-2} \mathbb{E}\left[H(1,1) \mathbb{1}_{\left\{|H(1,1)| \geq \frac{\varepsilon \theta_{n}^{2} n}{p}\right\}} \stackrel{\stackrel{n \rightarrow \infty}{\longrightarrow} 0,}{ }\right. \text {. }
\end{array}
$$

for any $\varepsilon>0$.

Proof. We use the definitions of $\tilde{\Phi}$ and $\tilde{G}$ :

(C1): We use Lemma A.8 for $l=2, \ldots, n$ and $a_{l}=\Psi_{l}(1)$. Then

$$
\begin{aligned}
\frac{1}{n \theta_{n}^{2}} \mathbb{E}\left[\left(\sum_{j=2}^{n} \Psi_{j}(1)\right)^{2} \mathbb{1}_{\left\{\left|\sum_{j=2}^{n} \Psi_{j}(1)\right| \geq \varepsilon \theta_{n} n\right\}}\right] & \leq \frac{1}{n \theta_{n}^{2}} \mathbb{E}\left[n^{2} \sum_{j=2}^{n} \Psi_{j}^{2}(1) \mathbb{1}_{\left\{\left|\Psi_{j}(1)\right| \geq \varepsilon \theta_{n}\right\}}\right] \\
& \leq \frac{n^{2}}{\theta_{n}^{2}} \mathbb{E}\left[\Psi_{2}^{2}(1) \mathbb{1}_{\left\{\left|\Psi_{2}(1)\right| \geq \varepsilon \theta_{n}\right\}}\right] \rightarrow 0
\end{aligned}
$$

by identical distribution and (C1"). Therefore, (C1) is true.

(C2): By Lemma A.8 for $k=3$,

$$
\begin{aligned}
\theta_{n}^{-2} \mathbb{E}\left[\tilde{\Phi}^{2}(1,2) \mathbb{1}_{\left\{|\tilde{\Phi}(1,2)| \geq \varepsilon \theta_{n} n\right\}}\right] \leq 9 \theta_{n}^{-2} \mathbb{E}\left[\Phi^{2}(1,2) \mathbb{1}_{\left\{|\Phi(1,2)| \geq \frac{\varepsilon \theta_{n} n}{3}\right\}}\right] \\
\quad+9 \theta_{n}^{-2} \mathbb{E}\left[\Psi_{2}^{2}(1) \mathbb{1}_{\left\{\left|\Psi_{2}(1)\right| \geq \frac{\varepsilon \theta_{n} n}{3}\right\}}\right]+9 \theta_{n}^{-2} \mathbb{E}\left[\Psi_{1}^{2}(2) \mathbb{1}_{\left\{\left|\Psi_{1}(2)\right| \geq \frac{\varepsilon \theta_{n} n}{3}\right\}}\right] \\
\leq 9 \theta_{n}^{-2} \mathbb{E}\left[\Phi^{2}(1,2) \mathbb{1}_{\left\{|\Phi(1,2)| \geq \frac{\varepsilon \theta_{n} n}{3}\right\}}\right] \\
\quad+9 \theta_{n}^{-2} \mathbb{E}\left[\Psi_{2}^{2}(1) \mathbb{1}_{\left\{\left|\Psi_{2}(1)\right| \geq \frac{\varepsilon \theta_{n}}{3}\right\}}\right]+9 \theta_{n}^{-2} \mathbb{E}\left[\Psi_{1}^{2}(2) \mathbb{1}_{\left\{\left|\Psi_{1}(2)\right| \geq \frac{\varepsilon \theta_{n}}{3}\right\}}\right]
\end{aligned}
$$


By (C2"), the first term converges to 0. By (C1"), so do the other two. Therefore, (C2) is true.

(C3): For $i \neq k$, we have

$$
\begin{aligned}
& \tilde{H}(i, i)=\mathbb{E}\left[\tilde{h}(i, k) \tilde{h}(i, k) \mid X_{i}\right] \\
&=\mathbb{E}\left[h(i, k) h(i, k)-\mathbb{E}\left[h(i, k) \mid X_{i}\right] h(i, k)-\mathbb{E}\left[h(i, k) \mid X_{k}\right] h(i, k)\right. \\
&-\mathbb{E}\left[h(i, k) \mid X_{i}\right] h(i, k)-\mathbb{E}\left[h(i, k) \mid X_{k}\right] h(i, k) \\
&+\mathbb{E}\left[h(i, k) \mid X_{i}\right] \mathbb{E}\left[h(i, k) \mid X_{i}\right]+\mathbb{E}\left[h(i, k) \mid X_{i}\right] \mathbb{E}\left[h(i, k) \mid X_{k}\right] \\
&\left.+\mathbb{E}\left[h(i, k) \mid X_{k}\right] \mathbb{E}\left[h(i, k) \mid X_{i}\right]+\mathbb{E}\left[h(i, k) \mid X_{k}\right] \mathbb{E}\left[h(i, k) \mid X_{k}\right] \mid X_{i}\right]
\end{aligned}
$$

By measurability and independence we obtain after a short computation

$$
\begin{aligned}
\tilde{H}(i, i)= & H(i, i)-\mathbb{E}\left[h(i, k) \mid X_{i}\right]^{2}-\mathbb{E}\left[\mathbb{E}\left[h(i, k) \mid X_{k}\right] h(i, k) \mid X_{i}\right] \\
& -\mathbb{E}\left[h(i, k) \mid X_{i}\right]^{2}-\mathbb{E}\left[\mathbb{E}\left[h(i, k) \mid X_{k}\right] h(i, k) \mid X_{i}\right] \\
& +\mathbb{E}\left[h(i, k) \mid X_{i}\right]^{2}+\mathbb{E}\left[h(i, k) \mid X_{i}\right] \mathbb{E}\left[\mathbb{E}\left[h(i, k) \mid X_{k}\right]\right] \\
& +\mathbb{E}\left[h(i, k) \mid X_{i}\right] \mathbb{E}\left[\mathbb{E}\left[h(i, k) \mid X_{k}\right]\right]+\mathbb{E}\left[\mathbb{E}\left[h(i, k) \mid X_{k}\right]^{2}\right]
\end{aligned}
$$

As $h(i, j)$ is centered, and by Lemma 1.1

$$
\begin{aligned}
& =H(i, i)-\mathbb{E}\left[h(i, k) \mid X_{i}\right]^{2}-2 \mathbb{E}\left[\mathbb{E}\left[h(i, k) \mid X_{k}\right] h(i, k) \mid X_{i}\right]+\frac{\gamma_{n}^{2}}{p} \\
& =: H(i, i)-A-2 B+\frac{\gamma_{n}^{2}}{p} .
\end{aligned}
$$

Firstly, by Lemma $1.1 \mathbb{E}[A]=\mathbb{E}\left[\mathbb{E}\left[h(i, k) \mid X_{k}\right]^{2}\right]=\frac{\gamma_{n}^{2}}{p}$.

By Cauchy-Schwarz and Lemma 1.1, we obtain

$$
\begin{aligned}
\left|\mathbb{E}\left[2 B \mathbb{1}_{\left\{|2 B| \geq \frac{\varepsilon \theta_{n}^{2} n}{5 p}\right\}}\right]\right| & \leq 2 \sqrt{\mathbb{E}\left[\mathbb{E}\left[h(i, k) \mid X_{k}\right]^{2}\right] \mathbb{E}\left[h(i, k)^{2} \mathbb{1}_{\left\{|2 B| \geq \frac{\varepsilon \theta_{n}^{2} n}{5 p}\right\}}\right.} \\
& \leq 2 \sqrt{\mathbb{E}\left[\mathbb{E}\left[h(i, k) \mid X_{k}\right]^{2}\right] \mathbb{E}\left[h(i, k)^{2}\right]} \leq 2 \frac{\gamma_{n} \beta_{n}}{p}
\end{aligned}
$$

We obtain by similar arguments as in Lemma A.8

$$
\begin{aligned}
& p \theta_{n}^{-2} \mathbb{E}\left[\tilde{H}(1,1) \mathbb{1}_{\left.\left\{|\tilde{H}(1,1)| \geq \frac{\varepsilon \theta_{n}^{2} n}{p}\right\}\right]}\right. \\
& \quad \leq 5 p \theta_{n}^{-2}\left(\mathbb{E}\left[H(1,1) \mathbb{1}_{\left\{|H(1,1)| \geq \frac{\varepsilon \theta_{5}^{2} n}{5 p}\right\}}\right]+\left|\mathbb{E}\left[A \mathbb{1}_{\left\{|A| \geq \frac{\varepsilon \theta_{n}^{2} n}{5 p}\right\}}\right]\right|+\left|\mathbb{E}\left[2 B \mathbb{1}_{\left\{|2 B| \geq \frac{\varepsilon \theta_{n}^{2} n}{5 p}\right\}}\right]\right|+\frac{\gamma_{n}^{2}}{p}\right) \\
& \quad \leq 5 p \theta_{n}^{-2}\left(\mathbb{E}\left[H(1,1) \mathbb{1}_{\left\{|H(1,1)| \geq \frac{\varepsilon \theta_{n}^{2} n}{5 p}\right\}}\right]+|\mathbb{E}[A]|+2 \frac{\gamma_{n} \beta_{n}}{p}+\frac{\gamma_{n}^{2}}{p}\right)
\end{aligned}
$$




$$
\begin{aligned}
& \leq 5 p \theta_{n}^{-2}\left(\mathbb{E}\left[H(1,1) \mathbb{1}_{\left\{|H(1,1)| \geq \frac{\varepsilon \theta_{n}^{2} n}{5 p}\right\}}\right]+2 \frac{\gamma_{n}^{2}}{p}+2 \frac{\gamma_{n} \beta_{n}}{p}\right) \\
& =5 p \theta_{n}^{-2} \mathbb{E}\left[H(1,1) \mathbb{1}_{\left\{|H(1,1)| \geq \frac{\varepsilon \theta_{n}^{2} n}{5 p}\right\}}\right]+10 p \theta_{n}^{-2} \frac{\gamma_{n}^{2}}{p}+10 p \theta_{n}^{-2} \frac{\beta_{n} \gamma_{n}}{p}
\end{aligned}
$$

Since $\theta_{n}^{2} \geq n p \gamma_{n}^{2}$ and $\theta_{n}^{2} \geq \frac{1}{2} \beta_{n}^{2}$, the last two terms immediately converge to 0 . By (C3"), so does the first one. Therefore, (C3) is true.

This completes the proof.

\section{A. Appendix}

We start the appendix by proving the lemmas in the introduction.

Proof of Lemma 1.1. We begin with (1)

$$
\begin{aligned}
\mathbb{E}\left[\tilde{\Phi}^{2}(i, j)\right] & =\mathbb{E}\left[\left(\Phi(i, j)-\Psi_{j}(i)-\Psi_{i}(j)\right)^{2}\right] \\
& =\mathbb{E}\left[\Phi^{2}(i, j)-2 \Psi_{j}(i) \Phi(i, j)-2 \Psi_{i}(j) \Phi(i, j)+\Psi_{j}^{2}(i)+2 \Psi_{j}(i) \Psi_{i}(j)+\Psi_{i}^{2}(j)\right] \\
& =\mathbb{E}\left[\Phi^{2}(i, j)\right]-4 \mathbb{E}\left[\Psi_{j}(i) \Phi(i, j)\right]+2 \mathbb{E}\left[\Psi_{j}^{2}(i)\right]+2 \mathbb{E}\left[\Psi_{j}(i) \Psi_{i}(j)\right]
\end{aligned}
$$

due to identical distribution. The last term is 0 , since $\Psi_{j}(i)$ and $\Psi_{i}(j)$ are independent and centered. The first and third term are known from (1.3). As for the second term,

$$
\begin{aligned}
\mathbb{E}\left[\Psi_{j}(i) \Phi(i, j)\right] & =\mathbb{E}\left[\mathbb{E}\left[\Phi(i, j) \mid X_{i}, Z_{i j}\right] \Phi(i, j)\right] \\
& =\mathbb{E}\left[\mathbb{E}\left[\mathbb{E}\left[\Phi(i, j) \mid X_{i}, Z_{i j}\right] \Phi(i, j) \mid X_{i}, Z_{i j}\right]\right] \\
& =\mathbb{E}\left[\mathbb{E}\left[\Phi(i, j) \mid X_{i}, Z_{i j}\right]^{2}\right]=\mathbb{E}\left[\Psi_{j}^{2}(i)\right]=\gamma_{n}^{2},
\end{aligned}
$$

by measurability, so that $\mathbb{E}\left[\tilde{\Phi}^{2}(i, j)\right]=\beta_{n}^{2}-4 \gamma_{n}^{2}+2 \gamma_{n}^{2}=\beta_{n}^{2}-2 \gamma_{n}^{2}$.

As for the statements on $h$ and $\tilde{h}$, i.e. (2): We have

$$
\gamma_{n}^{2}=\mathbb{E}\left[\Psi_{j}^{2}(i)\right]=\mathbb{E}\left[Z_{i, j}\right] \mathbb{E}\left[\mathbb{E}\left[h(i, j) \mid X_{i}\right]^{2}\right]=p \mathbb{E}\left[\mathbb{E}\left[h(i, j) \mid X_{i}\right]^{2}\right]
$$

by definition of $\Psi_{j}(i)$ and independence. Moreover,

$$
\beta_{n}^{2}=\mathbb{E}\left[\Phi^{2}(i, j)\right]=\mathbb{E}\left[Z_{i, j} h^{2}(i, j)\right]=\mathbb{E}\left[Z_{i, j}\right] \mathbb{E}\left[h^{2}(i, j)\right]=p \mathbb{E}\left[h^{2}(i, j)\right]
$$

by definition of $\Phi(i, j)$ and independence. Finally,

$$
\beta_{n}^{2}-2 \gamma_{n}^{2}=\mathbb{E}\left[\tilde{\Phi}^{2}(i, j)\right]=\mathbb{E}\left[Z_{i, j} \tilde{h}^{2}(i, j)\right]=\mathbb{E}\left[Z_{i, j}\right] \mathbb{E}\left[\tilde{h}^{2}(i, j)\right]=p \mathbb{E}\left[\tilde{h}^{2}(i, j)\right]
$$

by (11) and independence. This proves the claim. 
Proof of Lemma 1.2. We have

$$
\begin{aligned}
\mathcal{U}_{n} & =\left(\begin{array}{l}
n \\
2
\end{array}\right)^{-1} \sum_{1 \leq i<j \leq n} \tilde{\Phi}(i, j)+\left(\begin{array}{l}
n \\
2
\end{array}\right)^{-1} \sum_{1 \leq i<j \leq n} \Psi_{j}(i)+\left(\begin{array}{l}
n \\
2
\end{array}\right)^{-1} \sum_{1 \leq i<j \leq n} \Psi_{i}(j) \\
& =\left(\begin{array}{l}
n \\
2
\end{array}\right)^{-1} \sum_{1 \leq i<j \leq n} \tilde{\Phi}(i, j)+\left(\begin{array}{l}
n \\
2
\end{array}\right)^{-1} \sum_{1 \leq i<j \leq n} \Psi_{j}(i)+\left(\begin{array}{l}
n \\
2
\end{array}\right)^{-1} \sum_{1 \leq j<i \leq n} \Psi_{j}(i) \\
& =\left(\begin{array}{l}
n \\
2
\end{array}\right)^{-1}\left(\sum_{1 \leq i<j \leq n} \tilde{\Phi}(i, j)+\sum_{i=1}^{n} \sum_{\substack{j=1 \\
j \neq i}}^{n} \Psi_{j}(i)\right)=\left(\begin{array}{l}
n \\
2
\end{array}\right)^{-1}\left(\sum_{1 \leq j<i \leq n} \tilde{\Phi}(i, j)+\sum_{i=1}^{n} \sum_{\substack{j=1 \\
j \neq i}}^{n} \Psi_{j}(i)\right) .
\end{aligned}
$$

Proof of Lemma 1.3. Since $\mathcal{U}_{n}$ ist centered, we obtain from (1.5)

$$
\begin{aligned}
\mathbb{V} \mathcal{U}_{n}=\mathbb{E} & {\left[\mathcal{U}_{n}^{2}\right] } \\
= & \left(\begin{array}{l}
n \\
2
\end{array}\right)^{-2} \mathbb{E}\left[\sum_{i<j} \tilde{\Phi}^{2}(i, j)\right]+\left(\begin{array}{l}
n \\
2
\end{array}\right)^{-2} \mathbb{E}\left[\sum_{i<j} \sum_{\substack{k<l \\
\{i, j\}\{k, l\}}} \tilde{\Phi}(i, j) \tilde{\Phi}(k, l)\right] \\
& +2\left(\begin{array}{l}
n \\
2
\end{array}\right)^{-2} \mathbb{E}\left[\sum_{i<j} \tilde{\Phi}(i, j) \sum_{k \neq l} \Psi_{l}(k)\right] \\
& +\left(\begin{array}{l}
n \\
2
\end{array}\right)^{-2} \mathbb{E}\left[\sum_{j \neq i} \Psi_{j}^{2}(i)\right]+\left(\begin{array}{l}
n \\
2
\end{array}\right)^{-2} \mathbb{E}\left[\sum_{j \neq i} \sum_{\substack{l \neq k \\
\{i, j\} \neq\{k, l\}}} \Psi_{j}(i) \Psi_{l}(k)\right] \\
=: & A+B+C+D+E
\end{aligned}
$$

Let us consider the summands separately: Note that

$$
A=\left(\begin{array}{l}
n \\
2
\end{array}\right)^{-1} \mathbb{E}\left[\tilde{\Phi}^{2}(1,2)\right]=\left(\begin{array}{l}
n \\
2
\end{array}\right)^{-1}\left(\beta_{n}^{2}-2 \gamma_{n}^{2}\right) .
$$

Moreover, $B=C=0$ as follows from Lemma A.2. For $D$ notice that

$$
D=\left(\begin{array}{l}
n \\
2
\end{array}\right)^{-1} 2 \gamma_{n}^{2}
$$

Finally, consider $E$. For $k \neq i$, the expectation is 0 (see the arguments given in the proof of Lemma A.2). For $k=i$, we have that $j \neq l$ and therefore

$$
\begin{aligned}
\mathbb{E}\left[\Psi_{j}(i) \Psi_{l}(k)\right] & =\mathbb{E}\left[Z_{i j} Z_{i l} \mathbb{E}\left[h(i, j) \mid X_{i}\right] \mathbb{E}\left[h(i, l) \mid X_{i}\right]\right]=\mathbb{E}\left[Z_{i j} Z_{i l} \mathbb{E}\left[h(i, j) \mid X_{i}\right]^{2}\right] \\
& =\mathbb{E}\left[Z_{i l} \Psi_{j}^{2}(i)\right]=\mathbb{E}\left[Z_{i l}\right] \mathbb{E}\left[\Psi_{j}^{2}(i)\right]=p \gamma_{n}^{2}
\end{aligned}
$$

Thus

$$
E=\left(\begin{array}{l}
n \\
2
\end{array}\right)^{-1} \cdot 2(n-2) p \gamma_{n}^{2}
$$

and

$$
\mathbb{V} \mathcal{U}_{n}=\left(\begin{array}{l}
n \\
2
\end{array}\right)^{-1}\left(\beta_{n}^{2}+2(n-2) p \gamma_{n}^{2}\right) \sim\left(\begin{array}{l}
n \\
2
\end{array}\right)^{-1}\left(\beta_{n}^{2}+2 n p \gamma_{n}^{2}\right)=\left(\begin{array}{l}
n \\
2
\end{array}\right)^{-1} 2 \theta_{n}^{2}
$$


from which we conclude the assertion.

We now prove a couple of lemmas that were used in the proof of Theorem 1.5 in Section 4.

Corollary A.1. For any $i \neq j \mathbb{E}\left[\tilde{G}_{j}(i, i)\right]=\beta_{n}^{2}-2 \gamma_{n}^{2}$ and $\mathbb{E}[\tilde{H}(i, i)]=\frac{\beta_{n}^{2}-2 \gamma_{n}^{2}}{p}$.

Proof. The claim follows immediately from the tower property, the definition of $\tilde{G}$ and Lemma 1.1:

$$
\mathbb{E}\left[\tilde{G}_{j}(i, i)\right]=\mathbb{E}\left[\mathbb{E}\left[\tilde{\Phi}(i, j) \tilde{\Phi}(i, j) \mid X_{i}, Z_{i, j}\right]\right]=\mathbb{E}\left[\tilde{\Phi}^{2}(i, j)\right]=\beta_{n}^{2}-2 \gamma_{n}^{2} .
$$

For $\tilde{H}$, one can use

$$
p \mathbb{E}[\tilde{H}(i, i)]=\mathbb{E}\left[Z_{i, j}\right] \mathbb{E}[\tilde{H}(i, i)]=\mathbb{E}\left[Z_{i, j} \tilde{H}(i, i)\right]=\mathbb{E}\left[\tilde{G}_{j}(i, i)\right]
$$

and apply the above result.

Lemma A.2. For $\{i, j\} \neq\{k, l\}$ we have $\mathbb{E}[\tilde{\Phi}(i, j) \tilde{\Phi}(k, l)]=0$.

For any $\{i, j\},\{k, l\}$ we have $\mathbb{E}\left[\tilde{\Phi}(i, j) \Psi_{l}(k)\right]=0$.

Proof. Consider two cases:

If $\{i, j\} \cap\{k, l\}=1$, then by identical distributions and (1.4)

$$
\begin{aligned}
\mathbb{E}[\tilde{\Phi}(1,2) \tilde{\Phi}(1,3)] & =\mathbb{E}\left[Z_{12} Z_{13}\right] \mathbb{E}[\tilde{h}(1,2) \tilde{h}(1,3)] \\
& =\mathbb{E}\left[Z_{12} Z_{13}\right] \mathbb{E}\left[\mathbb{E}\left[\tilde{h}(1,2) \tilde{h}(1,3) \mid X_{1}, X_{2}\right]\right] \\
& =\mathbb{E}\left[Z_{12} Z_{13}\right] \mathbb{E}\left[\tilde{h}(1,2) \mathbb{E}\left[\tilde{h}(1,3) \mid X_{1}, X_{2}\right]\right] \\
& =\mathbb{E}\left[Z_{12} Z_{13}\right] \mathbb{E}\left[\tilde{h}(1,2) \mathbb{E}\left[\tilde{h}(1,3) \mid X_{1}\right]\right]=0
\end{aligned}
$$

If $\{i, j\} \cap\{k, l\}=0$, then by similar reasoning

$$
\mathbb{E}[\tilde{h}(1,2) \tilde{h}(3,4)]=0 \text {. }
$$

This shows the first claim. The second can be shown in the same fashion.

Lemma A.3. For $\Lambda_{j}(i)$ the following relation holds

$$
\mathbb{E}\left[\Lambda_{j}^{2}(i)\right]=O\left((n p)^{2}\right)
$$

Proof. With $\Lambda_{j}(i):=\sum_{\substack{k=1 \\ k \neq j}}^{i-1} Z_{i, k}+\sum_{\substack{k=i+1 \\ k \neq j}}^{n} p+1$ we can immediately conclude

$$
\begin{aligned}
\Lambda_{j}^{2}(i) & \leq 9 \cdot\left[\left(\sum_{k=1}^{i-1} Z_{i, k}\right)^{2}+((n-i) p)^{2}+1\right] \\
& =9 \cdot\left[\sum_{k=1}^{i-1} Z_{i, k}+\sum_{k=1}^{i-1} \sum_{\substack{l=1 \\
l \neq k}}^{i-1} Z_{i, k} Z_{i, l}+((n-i) p)^{2}+1\right]
\end{aligned}
$$


Therefore, using the independence of the $Z_{i, j}$

$$
\begin{aligned}
\mathbb{E}\left[\Lambda_{j}^{2}(i)\right] & \leq 9\left[\sum_{k=1}^{i-1} p+\sum_{k=1}^{i-1} \sum_{\substack{l=1 \\
l \neq k}}^{i-1} p^{2}+((n-i) p)^{2}+1\right] \\
& \leq 9\left[n p+n^{2} p^{2}+n^{2} p^{2}+1\right],
\end{aligned}
$$

which, due to $n p \rightarrow \infty$, confirms $\mathbb{E}\left[\Lambda_{j}^{2}(i)\right] \leq O\left((n p)^{2}\right)$.

Lemma A.4. With $\Lambda_{j}(i)$ as in Section 4 we have:

$$
\sum_{i=1}^{n} \sum_{i^{\prime}=1}^{n} \sum_{\substack{j=1 \\ j \neq i}}^{n} \sum_{\substack{j^{\prime}=1 \\ j^{\prime} \neq i^{\prime}}}^{n} \mathbb{E}\left[Z_{i, j} Z_{i^{\prime}, j^{\prime}} \Lambda_{j}(i) \Lambda_{j^{\prime}}\left(i^{\prime}\right)\right] \leq n^{4} p^{2}(n p)^{2}
$$

Proof. Recall that since $n p \rightarrow \infty$

$$
\Lambda_{j}(i):=\sum_{\substack{k=1 \\ k \neq j}}^{i-1} Z_{i, k}+\sum_{\substack{k=i+1 \\ k \neq j}}^{n} p+1 \quad \text { and } \quad \mathbb{E}\left[\Lambda_{j}(i)\right]=(n-2) p+1 \sim n p .
$$

Let us diffentiate cases.

If $i \neq i^{\prime}$ (and we're not in the case $i=j^{\prime}, j=i^{\prime}$, which will be considered later), then $\left(Z_{i, k}\right)_{\substack{k=1, \ldots, i-1 \\ k \neq j}}, Z_{i, j},\left(Z_{i^{\prime}, k}\right)_{\substack{k=1, \ldots, i^{\prime}-1 \\ k \neq j^{\prime}}}$ and $Z_{i^{\prime}, j^{\prime}}$ are independent (regardless of $j$ and $j^{\prime}$ ). Then by independence the expectation can be reduced to $\mathbb{E}\left[\Lambda_{j}(i)\right]^{2} \mathbb{E}\left[Z_{i j}\right]^{2} \sim(n p)^{2} p^{2}$ by (A.4). There are $n \cdot(n-1) \cdot((n-1) \cdot(n-1)-1)$ possibilities for this case.

If $i=j^{\prime}$ and $j=i^{\prime}$, the independence between $\Lambda_{j}(i), \Lambda_{i}(j)$ and $Z_{i, j}=Z_{j, i}=Z_{i^{\prime}, j^{\prime}}$ still holds, as well as the independence between $\Lambda_{j}(i)$ and $\Lambda_{i}(j)$ and we obtain

$$
\mathbb{E}\left[\Lambda_{j}(i) \Lambda_{j^{\prime}}\left(i^{\prime}\right) Z_{i, j} Z_{i^{\prime}, j^{\prime}}\right]=\mathbb{E}\left[\Lambda_{j}(i)\right] \mathbb{E}\left[\Lambda_{i}(j)\right] \mathbb{E}\left[Z_{i, j}^{2}\right] \sim(n p)^{2} p
$$

There are $n(n-1)$ possibilities for this case.

If $i=i^{\prime}$ but $j \neq j^{\prime}$, then $Z_{i, j}$ may appear in the random sum in $\Lambda_{j^{\prime}}(i)$ (and correspondingly, if we interchange $j, j^{\prime}$ ). We introduce

$$
\Lambda_{j, j^{\prime}}(i)=: \sum_{\substack{k=1 \\ k \neq j, j^{\prime}}}^{i-1} Z_{i, k}+\sum_{\substack{k=i+1 \\ k \neq j}}^{n} p+1, \quad \text { with } \mathbb{E}\left[\Lambda_{j, j^{\prime}}(i)\right]=(n-3) p+1 \sim n p
$$

and $\Lambda_{j}(i)=\Lambda_{j, j^{\prime}}(i)+Z_{i, j^{\prime}}$ Then

$$
\begin{aligned}
& \mathbb{E}\left[\Lambda_{j}(i) \Lambda_{j^{\prime}}\left(i^{\prime}\right) Z_{i, j} Z_{i^{\prime}, j^{\prime}}\right]=\mathbb{E}\left[\Lambda_{j}(i) \Lambda_{j^{\prime}}(i) Z_{i, j} Z_{i^{\prime}, j^{\prime}}\right] \\
& =\mathbb{E}\left[\Lambda_{j, j^{\prime}}^{2}(i) Z_{i, j} Z_{i, j^{\prime}}\right]+\mathbb{E}\left[Z_{i, j} Z_{i, j^{\prime}}\right]+2 \mathbb{E}\left[\Lambda_{j, j^{\prime}}(i) Z_{i, j} Z_{i, j^{\prime}}\right] \\
& =\mathbb{E}\left[\Lambda_{j, j^{\prime}}^{2}(i)\right] p^{2}+p^{2}+2 \mathbb{E}\left[\Lambda_{j, j^{\prime}}(i)\right] p^{2} .
\end{aligned}
$$

After some considerations one finds

$$
\mathbb{E}\left[\Lambda_{j, j^{\prime}}^{2}(i)\right]=O\left((n p)^{2}\right),
$$


so that combining this and (A.5) gives

$$
\mathbb{E}\left[\Lambda_{j}(i) \Lambda_{j^{\prime}}\left(i^{\prime}\right) Z_{i, j} Z_{i^{\prime}, j^{\prime}}\right] \leq O\left((n p)^{2}\right) p^{2}+p^{2}+2 O(n p) p^{2}=O\left((n p)^{2}\right) \cdot p^{2}
$$

by $n p \rightarrow \infty$. There are $n(n-1)(n-2)$ possibilities for the case $i=i^{\prime}, j \neq j^{\prime}$. If $i=i^{\prime}$ and $j=j^{\prime}$, we may again use independence to arrive at

$$
\mathbb{E}\left[\Lambda_{j}(i) \Lambda_{j^{\prime}}\left(i^{\prime}\right) Z_{i, j} Z_{i^{\prime}, j^{\prime}}\right]=\mathbb{E}\left[\Lambda_{j}^{2}(i) Z_{i, j}^{2}\right] \leq O\left((n p)^{2}\right) p,
$$

by Lemma A.3. Again, there are $n(n-1)$ possibilities for this case. Putting this together, we see that the sum of all expectations is asymptotically bounded from above by $n^{4}(n p)^{2} p^{2}$.

Lemma A.5. The following relation holds:

$$
\sum_{i=1}^{n} \sum_{\substack{j=1 \\ j \neq i}}^{n} \sum_{i^{\prime}=1}^{n} \sum_{j^{\prime}=1}^{i^{\prime}-1} \mathbb{E}\left[\Lambda_{j}(i) Z_{i, j} Z_{i^{\prime}, j^{\prime}}\right] \leq \frac{1}{2} n^{4} p^{2}(n p)
$$

Proof. The strategy of proof is exactly the same as in Lemma A.4, just the expectations and the number of summands differ. We therefore leave the proof to the reader.

Lemma A.6. Under the assumptions of Theorem 1.5 as $n \rightarrow \infty$ we have:

$$
\frac{1}{n^{4} \theta_{n}^{4}} \sum_{i=1}^{n} \sum_{\substack{j, k=1 \\ j \neq k}}^{i-1} \sum_{i^{\prime}=1}^{n} \sum_{\substack{j^{\prime}, k^{\prime}=1 \\ j^{\prime} \neq k^{\prime}}}^{i^{\prime}-1} \mathbb{E}\left[\tilde{\Phi}\left(i^{\prime}, j^{\prime}\right) \tilde{\Phi}\left(i^{\prime}, k^{\prime}\right) \tilde{\Phi}(i, j) \tilde{\Phi}(i, k)\right] \stackrel{n \rightarrow \infty}{\longrightarrow} 0 .
$$

Proof. Without loss of generality, assume $i^{\prime} \geq i$. We let

$$
Q:=\mathbb{E}\left[\tilde{G}_{i}(j, k) \tilde{G}_{i^{\prime}}\left(j^{\prime}, k^{\prime}\right)\right], \quad \tilde{Q}:=\mathbb{E}\left[\tilde{\Phi}(i, j) \tilde{\Phi}(i, k) \tilde{\Phi}\left(i^{\prime}, j^{\prime}\right) \tilde{\Phi}\left(i^{\prime}, k^{\prime}\right)\right]
$$

and $Q_{1} \cdot Q_{2}:=\mathbb{E}\left[Z_{i, j} Z_{i, k} Z_{i^{\prime}, j^{\prime}} Z_{i^{\prime}, k^{\prime}}\right] \mathbb{E}\left[\tilde{h}(i, j) \tilde{h}(i, k) \tilde{h}\left(i^{\prime}, j^{\prime}\right) \tilde{h}\left(i^{\prime}, k^{\prime}\right)\right]$. Note that all three notations denote the same object. However, we will use all these notations throughout the proof.

Now, let us go through all possible cases for $i, j, k, i^{\prime}, j^{\prime}, k^{\prime}$.

(1) If $i=i^{\prime}$ and $\left|\{j, k\} \cap\left\{j^{\prime}, k^{\prime}\right\}\right|=2$ by independence $Q=\mathbb{E}\left[\tilde{G}_{i}^{2}(j, k)\right]$.

(2) The cases $i=i^{\prime}$ and $\left|\{j, k\} \cap\left\{j^{\prime}, k^{\prime}\right\}\right|=1$ and $i=i^{\prime}$ and $\left|\{j, k\} \cap\left\{j^{\prime}, k^{\prime}\right\}\right|=0$

are almost identical. Consider the first: without loss of generality take $j=j^{\prime}$.

Then, by total expectation, the tower property, and independence

$$
\begin{aligned}
Q_{2} & =\mathbb{E}\left[\tilde{h}(i, j)^{2} \tilde{h}(i, k) \tilde{h}\left(i, k^{\prime}\right)\right]=\mathbb{E}\left[\mathbb{E}\left[\tilde{h}(i, j)^{2} \tilde{h}(i, k) \tilde{h}\left(i, k^{\prime}\right) \mid X_{i}, X_{j}, X_{k}\right]\right] \\
& =\mathbb{E}\left[\tilde{h}(i, j)^{2} \tilde{h}(i, k) \mathbb{E}\left[\tilde{h}\left(i, k^{\prime}\right) \mid X_{i}, X_{j}, X_{k}\right]\right]=\mathbb{E}\left[\tilde{h}(i, j)^{2} \tilde{h}(i, k) \mathbb{E}\left[\tilde{h}\left(i, k^{\prime}\right) \mid X_{i}\right]\right]=0,
\end{aligned}
$$

since by (1.4) $\mathbb{E}\left[\tilde{h}(l, m) \mid X_{r}\right]=0$ if $l \neq m$ for every $r$. Thus, $Q=0$. 
(3) Again the cases $i<i^{\prime}, i \in\left\{j^{\prime}, k^{\prime}\right\}$, and $\left|\{j, k\} \cap\left\{j^{\prime}, k^{\prime}\right\}\right|=1$ and $i<i^{\prime}$, $i \in\left\{j^{\prime}, k^{\prime}\right\}$, and $\left|\{j, k\} \cap\left\{j^{\prime}, k^{\prime}\right\}\right|=0$ are very similar. Consider the first: Without loss of generality $i=j^{\prime}, k=k^{\prime}$, and along the lines of the previous cases we get $Q_{2}=0$.

(4) Next consider the case $i<i^{\prime}, i \notin\left\{j^{\prime}, k^{\prime}\right\}$, and $\left|\{j, k\} \cap\left\{j^{\prime}, k^{\prime}\right\}\right|=2$. Without loss of generality, $j=j^{\prime}, k=k^{\prime}$ and by the definition of $\tilde{G}$ and independence we compute

$$
\begin{aligned}
Q & =\mathbb{E}\left[\tilde{G}_{i}(j, k) \tilde{G}_{i^{\prime}}(j, k)\right] \\
& =\mathbb{E}\left[\mathbb{E}\left[Z_{i, j} Z_{i, k} \tilde{h}(i, j) \tilde{h}(i, k) \mid X_{j}, X_{k}, Z_{i, j} Z_{i, k}\right] \mathbb{E}\left[Z_{i^{\prime}, j} Z_{i^{\prime}, k} \tilde{h}\left(i^{\prime}, j\right) \tilde{h}\left(i^{\prime}, k\right) \mid X_{j}, X_{k}, Z_{i^{\prime}, j} Z_{i^{\prime}, k}\right]\right] \\
& =\mathbb{E}\left[Z_{i, j} Z_{i, k} Z_{i^{\prime}, j} Z_{i^{\prime}, k} \mathbb{E}\left[\tilde{h}(i, j) \tilde{h}(i, k) \mid X_{j}, X_{k}\right]^{2}\right] \\
& =\mathbb{E}\left[Z_{i^{\prime}, j} Z_{i^{\prime}, k}\left(Z_{i, j} Z_{i, k} \mathbb{E}\left[\tilde{h}(i, j) \tilde{h}(i, k) \mid X_{j}, X_{k}, Z_{i, j} Z_{i, k}\right]\right)^{2}\right] \\
& =\mathbb{E}\left[Z_{i^{\prime}, j} Z_{i^{\prime}, k}\left(\tilde{G}_{i}(j, k)\right)^{2}\right]=p^{2} \mathbb{E}\left[\tilde{G}_{i}^{2}(j, k)\right]
\end{aligned}
$$

(5) Finally, the cases the case $i<i^{\prime}, i \notin\left\{j^{\prime}, k^{\prime}\right\}$, and $\left|\{j, k\} \cap\left\{j^{\prime}, k^{\prime}\right\}\right|=0,1$ follow the arguments in cases (2) and (3) to give $Q_{2}=0$.

To sum up, what we get from this case distinction: The only situation where the given expectation is non-zero is when $\left|\{j, k\} \cap\left\{j^{\prime}, k^{\prime}\right\}\right|=2$. In the case $i=i^{\prime}$, there are at most $n(n-1)^{2}$ possibilities for this ( $n$ for $i$, and since $j$ and $k$ are smaller than $i$ and different, at most $n-1$ for each of those). In the case $i \neq i^{\prime}$, there are an additional $n-1$ possibilities for $i^{\prime}$, which makes at most $n(n-1)^{3}$ possibilities. Altogether, we have that the given sum of expectations is bounded by

$$
n(n-1)^{2} \mathbb{E}\left[\left(\tilde{G}_{i}(j, k)\right)^{2}\right]+n(n-1)^{3} p^{2} \mathbb{E}\left[\left(\tilde{G}_{i}(j, k)\right)^{2}\right] \leq n^{4} \mathbb{E}\left[\tilde{G}_{i}^{2}(j, k)\right] .
$$

Then for the sum of the considered expectations we have

$$
\frac{1}{n^{4} \theta_{n}^{4}} \sum_{i=1}^{n} \sum_{\substack{j, k=1 \\ j \neq k}}^{i-1} \sum_{\substack{i^{\prime}=1 \\ j^{\prime}, k^{\prime}=1 \\ j^{\prime} \neq k^{\prime}}}^{n} \mathbb{E}\left[\tilde{\Phi}\left(i^{\prime}, j^{\prime}\right) \tilde{\Phi}\left(i^{\prime}, k^{\prime}\right) \tilde{\Phi}(i, j) \tilde{\Phi}(i, k)\right] \leq \frac{\mathbb{E}\left[\tilde{G}_{i}^{2}(j, k)\right]}{\theta_{n}^{4}}
$$

By (C4), this converges to 0 .

Lemma A.7. Under the assumptions of Theorem 1.5 as $n \rightarrow \infty$ we have

$$
\begin{aligned}
\frac{1}{n^{4} \theta_{n}^{4}} \sum_{i=1}^{n} \sum_{\substack{j=1 \\
j \neq i}}^{n} \sum_{m=1}^{i-1} \sum_{i^{\prime}=1}^{n} \sum_{\substack{j^{\prime}=1 \\
j^{\prime} \neq i^{\prime}}}^{n} \sum_{m^{\prime}=1}^{i^{\prime}-1} \mathbb{E}\left[\mathbb{E}\left[\Psi_{j}(i) \tilde{\Phi}(i, m) \mid X_{m},\left(Z_{l, i}\right)_{l=1, \ldots, i-1}\right]\right. \\
\left.\cdot \mathbb{E}\left[\Psi_{j^{\prime}}\left(i^{\prime}\right) \tilde{\Phi}\left(i^{\prime}, m^{\prime}\right) \mid X_{m^{\prime}},\left(Z_{l, i^{\prime}}\right)_{l=1, \ldots, i^{\prime}-1}\right]\right] \stackrel{n \rightarrow \infty}{\longrightarrow} 0 .
\end{aligned}
$$

Proof. We denote

$Q:=\mathbb{E}\left[\Psi_{j}(i) \tilde{\Phi}(i, m) \mid X_{m},\left(Z_{l, i}\right)_{l=1, \ldots, i-1}\right] \mathbb{E}\left[\Psi_{j}\left(i^{\prime}\right) \tilde{\Phi}\left(i^{\prime}, m^{\prime}\right) \mid X_{m^{\prime}},\left(Z_{l, i^{\prime}}\right)_{l=1, \ldots, i^{\prime}-1}\right]=Q_{1} \cdot Q_{2}$, 
where

$$
\begin{aligned}
Q_{1} & =\mathbb{E}\left[Z_{i, j} Z_{i, m} \mid\left(Z_{l, i}\right)_{l=1, \ldots, i-1}\right] \cdot \mathbb{E}\left[Z_{i^{\prime}, j^{\prime}} Z_{i^{\prime}, m^{\prime}} \mid\left(Z_{l, i^{\prime}}\right)_{l=1, \ldots, i^{\prime}-1}\right] \\
Q_{2} & =\mathbb{E}\left[\mathbb{E}\left[h(i, j) \mid X_{i}\right] \tilde{h}(i, m) \mid X_{m}\right] \mathbb{E}\left[\mathbb{E}\left[h\left(i^{\prime}, j^{\prime}\right) \mid X_{i^{\prime}}\right] \tilde{h}\left(i^{\prime}, m^{\prime}\right) \mid X_{m^{\prime}}\right] .
\end{aligned}
$$

By independence between the $Z$ - and $X$-terms, $\mathbb{E}[Q]=\mathbb{E}\left[Q_{1}\right] \mathbb{E}\left[Q_{2}\right]$.

In the case $m \neq m^{\prime}$, we have $\mathbb{E}\left[Q_{2}\right]=0$ by independence of $X_{m}$ and $X_{m}^{\prime}$ and $\mathbb{E}\left[\mathbb{E}\left[h(i, j) \mid X_{i}\right] \tilde{h}(i, m)\right]=0$, which we find by adding a conditional expectation on $X_{i}$.

For $m=m^{\prime}$, note that the conditional expectations in $Q_{2}$ do not depend on the choice of $i$ and $i^{\prime}$, hence we choose $i=1, i^{\prime}=2$, and $m=3$. Then:

$$
\begin{aligned}
\mathbb{E}\left[Q_{2}\right] & =\mathbb{E}\left[\mathbb{E}\left[\mathbb{E}\left[h(1, j) \mid X_{1}\right] \tilde{h}(1,3) \mid X_{3}\right] \mathbb{E}\left[\mathbb{E}\left[h\left(2, j^{\prime}\right) \mid X_{2}\right] \tilde{h}(2,3) \mid X_{3}\right]\right] \\
& =\mathbb{E}\left[\mathbb{E}\left[h(1, j) \mid X_{1}\right] \mathbb{E}\left[h\left(2, j^{\prime}\right) \mid X_{2}\right] \tilde{H}_{3}(1,2)\right] .
\end{aligned}
$$

By Cauchy-Schwarz, independence and Lemma 1.1

$\mathbb{E}\left[Q_{2}\right] \leq\left(\mathbb{E}\left[\mathbb{E}\left[h(1, j) \mid X_{1}\right]^{2}\right] \mathbb{E}\left[\mathbb{E}\left[h\left(2, j^{\prime}\right) \mid X_{2}\right]^{2}\right] \mathbb{E}\left[\tilde{H}_{3}^{2}(1,2)\right]\right)^{1 / 2}=\frac{\gamma_{n}^{2}}{p} \mathbb{E}\left[\tilde{H}_{3}^{2}(1,2)\right]^{1 / 2}$

Furthermore, if $i=i^{\prime}$ and $\left|\left\{j, j^{\prime}, m\right\}\right| \leq 2, \mathbb{E}\left[Q_{1}\right] \leq p$ and we have a at most $n^{3}$ possibilities to choose $i, j, m, i^{\prime}, j^{\prime}, m^{\prime}$.

If $i=i^{\prime}$ and $\left|\left\{j, j^{\prime}, m\right\}\right|=3, \mathbb{E}\left[Q_{1}\right] \leq p^{3}$ and at most $n^{4}$ possibilities to choose.

If $i \neq i^{\prime}$ and $\left\{j, j^{\prime}, m\right\} \mid \leq 2, \mathbb{E}\left[Q_{1}\right] \leq p^{2}$ and we have at most $n^{4}$ possibilities to choose.

If $i \neq i^{\prime}$ and $\left\{j, j^{\prime}, m\right\} \mid=3, \mathbb{E}\left[Q_{1}\right] \leq p^{4}$ and we have at most $n^{5}$ possibilities to choose.

Combining all this and keeping in mind that we assume that $n p \rightarrow \infty$ yields

$$
\begin{gathered}
\frac{1}{n^{4} \theta_{n}^{4}} \sum_{i=1}^{n} \sum_{\substack{j=1 \\
j \neq i}}^{n} \sum_{m=1}^{i-1} \sum_{\substack{i^{\prime}=1 \\
j^{\prime}=1}}^{n} \sum_{\substack{j^{\prime} \\
j^{\prime}}}^{n} \sum_{m^{\prime}=1}^{i^{\prime}-1} \mathbb{E}\left[\mathbb{E}\left[\Psi_{j}(i) \tilde{\Phi}(i, m) \mid X_{m},\left(Z_{l, i}\right)_{l=1, \ldots, i-1}\right]\right. \\
\left.\cdot \mathbb{E}\left[\Psi_{j^{\prime}}\left(i^{\prime}\right) \tilde{\Phi}\left(i^{\prime}, m^{\prime}\right) \mid X_{m^{\prime}},\left(Z_{l, i^{\prime}}\right)_{l=1, \ldots, i^{\prime}-1}\right]\right] \\
\leq \frac{1}{n^{4} \theta_{n}^{4}} \frac{\gamma_{n}^{2}}{p} \sqrt{\mathbb{E}\left[\tilde{H}_{1}^{2}(2,3)\right]}\left(p n^{3}+p^{3} n^{4}+p^{2} n^{4}+p^{4} n^{5}\right) \\
\leq \frac{2}{n^{4} \theta_{n}^{4}} \frac{\gamma_{n}^{2}}{p} \sqrt{\mathbb{E}\left[\tilde{H}_{1}^{2}(2,3)\right]}\left(p^{2} n^{4}+p^{4} n^{5}\right)
\end{gathered}
$$




$$
\begin{aligned}
& \leq \frac{2}{n p} \sqrt{\frac{1}{\theta_{n}^{4}} p^{2} \mathbb{E}\left[\tilde{H}_{1}^{2}(2,3)\right]}+8 p \sqrt{\frac{1}{\theta_{n}^{4}} p^{2} \mathbb{E}\left[\tilde{H}_{1}^{2}(2,3)\right]} \\
& \leq \frac{2}{n p} \sqrt{\frac{1}{\theta_{n}^{4}} \mathbb{E}\left[\tilde{G}_{1}^{2}(2,3)\right]}+8 p \sqrt{\frac{1}{\theta_{n}^{4}} \mathbb{E}\left[\tilde{G}_{1}^{2}(2,3)\right]} .
\end{aligned}
$$

where the last two inequalities follow from (1.3) and (1.7). By $n p \rightarrow \infty$ and (C4), this converges to 0 .

A very general lemma for the purpose of reminding us of a basic fact is

Lemma A.8. For any sequence $\left(a_{l}\right)_{l=1, \ldots, k}$, the following relation holds

$$
\left(\sum_{l=1}^{k} a_{l}\right)^{2} \mathbb{1}_{\left\{\left|\sum_{l=1}^{k} a_{l}\right| \geq \varepsilon\right\}} \leq k^{2} \sum_{l=1}^{k} a_{l}^{2} \mathbb{1}_{\left\{\left|a_{l}\right| \geq \frac{\varepsilon}{k}\right\}}
$$

The proof of this lemma is elementary.

\section{REFERENCES}

[BG95] V. Bentkus and F. Götze. On minimal moment assumptions in Berry-Esséen theorems for $U$-statistics. Teor. Veroyatnost. i Primenen., 40(3):596-614, 1995.

[BGvZ86] P. J. Bickel, F. Götze, and W. R. van Zwet. The Edgeworth expansion for $U$-statistics of degree two. Ann. Statist., 14(4):1463-1484, 1986.

[Chr92] Tasos C. Christofides. A strong law of large numbers for $U$-statistics. J. Statist. Plann. Inference, 31(2):133-145, 1992.

[Den85] Manfred Denker. Asymptotic distribution theory in nonparametric statistics. Advanced Lectures in Mathematics. Friedr. Vieweg \& Sohn, Braunschweig, 1985.

[Eic98] Peter Eichelsbacher. Moderate and large deviations for $U$-processes. Stochastic Process. Appl., 74(2):273-296, 1998.

[EL95] Peter Eichelsbacher and Matthias Löwe. A large deviation principle for $m$-variate von Mises-statistics and $U$-statistics. J. Theoret. Probab., 8(4):807-824, 1995.

[EL98] P. Eichelsbacher and M. Löwe. Large deviations principle for partial sums $U$-processes. Teor. Veroyatnost. i Primenen., 43(1):97-115, 1998.

[Gir90] V. L. Girko. Theory of Random Determinants, volume 45 of Mathematics and Its Applications. Kluwer Academic Publishers, Dordrecht, 1990.

[Hoe48] Wassily Hoeffding. A class of statistics with asymptotically normal distribution. Ann. Math. Statistics, 19:293-325, 1948.

[Jan84] Svante Janson. The asymptotic distributions of incomplete $U$-statistics. Z. Wahrsch. Verw. Gebiete, 66(4):495-505, 1984.

[Jan90] Svante Janson. A functional limit theorem for random graphs with applications to subgraph count statistics. Random Structures Algorithms, 1(1):15-37, 1990.

[JJ86] S. Rao Jammalamadaka and Svante Janson. Limit theorems for a triangular scheme of $U$-statistics with applications to inter-point distances. Ann. Probab., 14(4):1347-1358, 1986.

[Lee90] A. J. Lee. U-statistics, volume 110 of Statistics: Textbooks and Monographs. Marcel Dekker, Inc., New York, 1990. Theory and practice.

[LT20] Matthias Löwe and Sara Terveer. A central limit theorem for the mean starting hitting time for a random walk on a random graph. in preparation, 2020. 
[MA87] T. L. Malevich and G. R. Abdurakhmanov. On conditions for the asymptotic normality of $U$-statistics in the case of triangular arrays. Izv. Akad. Nauk UzSSR Ser. Fiz.-Mat. Nauk, 2:21-28, 78, 1987.

(Matthias Löwe) Fachbereich Mathematik und Informatik, Universität Münster, EinSteinstrasse 62, 48149 MÜnster, Germany

E-mail address, Matthias Löwe: maloewe@uni-muenster.de

(Sara Terveer) Fachbereich Mathematik und Informatik, Universität MÜnster, EinSteinstrasse 62, 48149 Münster, Germany

E-mail address, Sara Terveer: sara.terveer@uni-muenster.de 